\title{
VLT spectroscopy of NGC 3115 globular clusters ${ }^{\star}$
}

\author{
H. Kuntschner ${ }^{1,4}$, B. L. Ziegler, ${ }^{2,3}$, R. M. Sharples ${ }^{4}$, G. Worthey ${ }^{5}$, and K. J. Fricke ${ }^{3}$ \\ ${ }^{1}$ European Southern Observatory, Karl-Schwarzschild-Str. 2, 85748 Garching bei München, Germany \\ 2 Academy of Sciences, Theaterstr. 7, 37073 Göttingen, Germany \\ 3 Universitätssternwarte, Geismarlandstrasse 11, 37083 Göttingen, Germany \\ ${ }^{4}$ Department of Physics, University of Durham, Durham DH1 3LE, UK \\ 5 Department of Physics, Washington State University, 1245 Webster Hall, Pullman, WA 99164-2814, USA
}

Received 17 July 2002 / Accepted 9 September 2002

\begin{abstract}
We present results derived from VLT-FORS2 spectra of 24 different globular clusters associated with the lenticular galaxy NGC 3115. A subsample of 17 globular clusters have sufficiently high signal-to-noise to allow precision measurements of absorption line-strengths. Comparing these indices to new stellar population models by Thomas et al. we determine ages, metallicities and element abundance ratios. For the first time these stellar population models explicitly take abundance ratio biases in the Lick/IDS stellar library into account. Our data are also compared with the Lick/IDS observations of Milky Way and M 31 globular clusters. Unpublished higher order Balmer lines $\left(\mathrm{H} \gamma_{\mathrm{A}, \mathrm{F}}\right.$ and $\left.\mathrm{H} \delta_{\mathrm{A}, \mathrm{F}}\right)$ from the Lick/IDS observations are given in the Appendix.

Our best age estimates show that the observed clusters which sample the bimodal colour distribution of NGC 3115 are coeval within our observational errors (2-3 Gyr). Our best calibrated age/metallicity diagnostic diagram $(\mathrm{H} \beta \mathrm{vs}$. $[\mathrm{MgFe}])$ indicates an absolute age of 11-12 Gyr consistent with the luminosity weighted age for the central part of NGC 3115 . We confirm with our accurate line-strength measurements that the $(V-I)$ colour is a good metallicity indicator within the probed metallicity range $(-1.5<[\mathrm{Fe} / \mathrm{H}]<0.0)$. The abundance ratios for globular clusters in NGC 3115 give an inhomogeneous picture. We find a range from solar to super-solar ratios for both blue and red clusters. This is similar to the data for M 31 while the Milky Way seems to harbour clusters which are mainly consistent with $[\alpha / \mathrm{Fe}] \simeq 0.3$. From our accurate recession velocities we detect, independent of metallicity, clear rotation in the sample of globular clusters.

In order to explain the metallicity and abundance ratio pattern, particularly the range in abundance ratios for the metal rich globular clusters in NGC 3115, we favour a formation picture with more than two distinct formation episodes.
\end{abstract}

Key words. galaxies: abundances - galaxies: individual: NGC 3115 - galaxies: star clusters - galaxies: stellar content globular clusters: general

\section{Introduction}

The analysis of globular cluster systems in external galaxies is starting to fulfil its long-held promise as a probe of the formation of galaxies. Globular clusters (hereafter GCs) are a fossil record of this formation process, and provide one of the best tools with which to investigate the chemical enrichment and star formation history in the initial stages of galaxy formation (e.g., Ashman \& Zepf 1998). Much recent interest has focused on the globular cluster systems of luminous elliptical galaxies where the combination of metallicities and kinematics can be used to distinguish between variants of the popular monolithic collapse and merger models for galaxy formation (e.g., Forbes et al. 1997; Sharples et al. 1998).

Send offprint requests to: $\mathrm{H}$. Kuntschner,

e-mail: hkuntsch@eso.org

* Based on observations collected at the European Southern Observatory, Cerro Paranal, Chile (ESO No. 66.B-0131).
Lenticular galaxies hold a key position in the Hubble sequence of morphological types, intermediate between pure spheroidal systems like luminous ellipticals and diskdominated spiral galaxies. Their formation mechanism is still the subject of considerable debate with evidence both for (Dressler et al. 1997) and against (Dressler 1980) their evolution from star-forming spirals via processes of gas stripping and exhaustion. A key question is when and how did such processes occur for $\mathrm{S} 0$ galaxies in a wide range of environments from rich clusters to the field. The globular cluster systems of S0 galaxies can provide independent constraints on when the major star formation episodes occurred both in the disk and halo. However, thus far they have been little studied with only NGC 1380 (Kissler-Patig et al. 1997) and NGC 4594 (the Sombrero galaxy, Bridges et al. 1997) having received any detailed attention and only with photometric methods. 
NGC 3115 is one of the nearest S0 galaxies $(9.7 \pm 0.4 \mathrm{Mpc}$, $M_{B}=-20.1$; Tonry et al. 2001) and is located in the sparse low-density environment of the Leo Spur. As such it provides an ideal test case for studying the formation mechanism of field S0's. A significant globular cluster system containing $~ 500$ clusters was first detected by Hanes \& Harris (1986) using photographic plates. The nature of the cluster system and its origin were recently thrown into question with the discovery by Elson (1997) that the red-giant stars in the NGC 3115 halo $\sim 40 \mathrm{kpc}$ from the centre showed a bimodal colour distribution. The inferred presence of two distinct halo populations of roughly equal size at metallicities of $[\mathrm{Fe} / \mathrm{H}] \simeq-0.7$ and $[\mathrm{Fe} / \mathrm{H}] \simeq-1.3$ suggests at least two distinct epochs of formation.

The $(V-I)$ colour distribution of the NGC 3115 globular cluster system has been the target of two recent independent studies using HST (Kundu \& Whitmore 1998) and CFHT (Kavelaars 1998) data. Both studies find bimodality in the colour distributions of the GCs, with mean metallicities at $[\mathrm{Fe} / \mathrm{H}] \simeq-0.37$ and $[\mathrm{Fe} / \mathrm{H}] \simeq-1.36$ suggesting that the cluster and halo star systems may have formed coevally. This suggestion has gained further support from an investigation by Puzia et al. (2002b) who employed optical-IR colours to probe the globular cluster population close to the centre of NGC 3115. They also find two peaks in metallicity and an average age around $\approx 10 \mathrm{Gyr}$. However, their age discrimination power is very limited for metallicities lower than $[\mathrm{Fe} / \mathrm{H}]=-0.4$.

One scenario in which the above observations could be understood is if the metal-poor component corresponds to a primordial $\simeq 13$ Gyr old population, whilst the metal-rich component formed a few Gyr later from enriched gas, possibly as the result of a minor merger (e.g., Bekki 1998). With only broadband colours available, however, the well-known degeneracy between metallicity and age (Worthey 1994) makes such conclusions very uncertain.

For that reason, we have started a campaign to spectroscopically study the globular cluster system in NGC 3115. Our precision measurements of absorption line-strengths can be used to derive age and metallicity estimates directly from the comparison with new stellar population models. Unlike photometric methods, with spectroscopy we are also able to explore element abundance ratios for the GCs. We compare our results with other very recently obtained spectroscopic samples of GCs in early-type galaxies: in the giant Fornax elliptical NGC 1399 (Forbes et al. 2001), the Sa/Sb galaxy M 81 (Schroder et al. 2002), the SB0 galaxy NGC 1023 (Larsen $\&$ Brodie 2002) and the Sombrero galaxy NGC 4594 (Larsen et al. 2002).

The paper is organized as follows. In Sect. 2, the observations and their reduction are discussed. Section 3 presents the colour distribution of our sample while in Sect. 4 the treatment of abundance ratios and new stellar population models are investigated. Our results on abundance ratios, age and metallicity distributions for GCs in NGC 3115, the Milky Way (hereafter MW) and M 31 are presented in Sect. 5 with a general discussion in Sect. 6. We present our conclusions in Sect. 7.

\section{Observations and data reduction}

The candidate GCs were selected from the HST/WFPC2 investigation of Kundu \& Whitmore (1998) who detected 144 globular cluster candidates in the central region of NGC 3115. In order to keep integration times reasonably short only clusters with $V<22$ (the peak of the GC luminosity function is at $V=22.37 \pm 0.05$; Kundu \& Whitmore 1998) were selected while keeping a balance between red $((V-I)<1.06)$ and blue $((V-I) \geq 1.06)$ clusters. In order to utilize the full field-ofview of our multi-object spectrograph we supplemented this list with GC candidates from a low resolution spectroscopy survey reported in Kavelaars (1998) and also placed some slits on promising objects without prior information (see Table 1). In total two masks with each 26 slitlets covering more than 50 different GC candidates were manufactured.

The observations were carried out 26/27 Feb. 2001 with FORS2 on VLT using the blue $600 \mathrm{l} / \mathrm{mm}$ grism and 1" wide slitlets giving a resolution of $\sim 4.8 \AA(F W H M)$ sampled at $1.2 \AA_{\text {pixel }^{-1}}$. The seeing was generally $F W H M \leq 11^{\prime \prime} 0$. The total exposure time for the first mask was $12440 \mathrm{~s}$ split up in six individual exposures of varying length. Due to bad weather the second mask was only exposed for $2700 \mathrm{~s}$ which was not long enough to be of use for this study. The flux standard GD 71 was observed through a long-slit ( 2 !' 5 width) to enable us to correct the continuum shape of the spectra.

The standard data reduction procedures (bias subtraction, flat-fielding, wavelength calibration, sky-subtraction and continuum correction to a relative flux scale) were performed with a combination of MIDAS and IRAF tasks. For each slit a 2-dimensional subsection of the CCD was extracted and then treated as a long-slit observation. The extraction region was defined by tracing the globular cluster spectrum along the wavelength direction and extracting the corresponding sections for the flat-field and arc-lamp observations. The cosmic rays were removed with the routine lacos_spec (van Dokkum 2001). The wavelength calibration was accurate to $<0.2 \AA$. After skysubtraction GCs were extracted from the individual exposures to achieve an optimal $S / N$ (Horne 1986). Finally, the spectra of the individual exposures were combined.

We used the spectroscopic flux standard GD 71 to correct the continuum shape of our spectra to a relative flux scale and applied a reddening correction of $E(B-V)=0.146$ (Schlegel et al. 1998). In order to transform our observations onto the Lick/IDS system we convolved our spectra with a wavelengthdependent Gaussian kernel (taking into account small variations of the instrumental resolution with position on the chip) thereby reproducing the Lick/IDS spectral resolution (Worthey \& Ottaviani 1997).

As a first analysis step we measured the recession velocity (with fxcor in IRAF) and $S / N$ of the GC candidates. The first slit (Slitlet ID: 1) did not show any object which we ascribe to a field distortion towards the edges of the field-of-view. Basic information for the remaining 28 globular cluster candidates is listed in Table 1. The first column in this table is an object identifier, the second column indicates from which source the object was selected from. The following columns list the J2000.0 coordinates and $V, I$ photometry of Kundu \& Whitmore (1998). 
Table 1. List of observed globular cluster candidates.

\begin{tabular}{|c|c|c|c|c|c|c|c|c|c|}
\hline \multirow{2}{*}{$\frac{\text { Slitlet ID }}{1}$} & \multirow{2}{*}{$\underbrace{\text { Origin }^{b}}_{-}$} & \multicolumn{2}{|c|}{ RA J2000.0 Dec } & $V(\mathrm{HST})$ & $(V-I)(\mathrm{HST})$ & \multirow[t]{2}{*}{$(V-I)(\mathrm{spec})^{c}$} & \multirow[t]{2}{*}{$c z$} & \multirow[t]{2}{*}{$S / N$} & \multirow[t]{2}{*}{ member } \\
\hline & & & & No & bject in slit. & & & & \\
\hline 2 & - & $10^{\mathrm{h}} 05^{\mathrm{m}} 06^{\prime \prime} 5$ & $-7^{\circ} 45^{\mathrm{m}} 01^{\prime \prime}$ & - & - & 1.183 & $408 \pm 37$ & 9 & $\sqrt{ }$ \\
\hline 3 & - & $10^{\mathrm{h}} 05^{\mathrm{m}} 06^{\prime \prime} 1$ & $-7^{\circ} 44^{\mathrm{m}} 30^{\prime \prime}$ & - & - & 1.142 & $439 \pm 17$ & 23 & $\sqrt{ }$ \\
\hline 4 & - & $10^{\mathrm{h}} 05^{\mathrm{m}} 09^{\prime} 9$ & $-7^{\circ} 45^{\mathrm{m}} 01^{\prime \prime}$ & - & - & 0.883 & $0 \pm 11$ & 72 & - \\
\hline 5 & WHT09 & $10^{\mathrm{h}} 05^{\mathrm{m}} 11^{\prime \prime} .4$ & $-7^{\circ} 45^{\mathrm{m}} 01^{\prime \prime}$ & - & - & 0.980 & $379 \pm 21$ & 32 & $\sqrt{ }$ \\
\hline 6 & - & $10^{\mathrm{h}} 05^{\mathrm{m}} 09^{\prime} .2$ & $-7^{\circ} 43^{\mathrm{m}} 52^{\prime \prime}$ & - & - & - & $z=0.39^{a}$ & - & - \\
\hline 7 & HST08 & $10^{\mathrm{h}} 05^{\mathrm{m}} 12^{\prime \prime} .6$ & $-7^{\circ} 44^{\mathrm{m}} 28^{\prime \prime}$ & $20.282 \pm 0.006$ & $1.178 \pm 0.008$ & 1.148 & $291 \pm 16$ & 28 & $\sqrt{ }$ \\
\hline 8 & HST03 & $10^{\mathrm{h}} 05^{\mathrm{m}} 12^{\prime \prime} 6$ & $-7^{\circ} 44^{\mathrm{m}} 09^{\prime \prime}$ & $19.794 \pm 0.006$ & $1.051 \pm 0.009$ & 1.029 & $393 \pm 13$ & 61 & $\sqrt{ }$ \\
\hline 9 & HST02 & $10^{\mathrm{h}} 05^{\mathrm{m}} 13^{\prime \prime} 1$ & $-7^{\circ} 43^{\mathrm{m}} 59^{\prime \prime}$ & $19.742 \pm 0.005$ & $1.077 \pm 0.007$ & 1.068 & $494 \pm 16$ & 38 & $\sqrt{ }$ \\
\hline 10 & HST21 & $10^{\mathrm{h}} 05^{\mathrm{m}} 13^{\prime \prime} 0$ & $-7^{\circ} 43^{\mathrm{m}} 41^{\prime \prime}$ & $21.072 \pm 0.012$ & $0.979 \pm 0.018$ & 1.050 & $456 \pm 37$ & 8 & $\sqrt{ }$ \\
\hline 11 & HST39 & $10^{\mathrm{h}} 05^{\mathrm{m}} 14^{\prime \prime} \cdot 7$ & $-7^{\circ} 43^{\mathrm{m}} 56^{\prime \prime}$ & $21.539 \pm 0.014$ & $1.199 \pm 0.018$ & 1.218 & $702 \pm 31$ & 8 & $\sqrt{ }$ \\
\hline 12 & HST13 & $10^{\mathrm{h}} 05^{\mathrm{m}} 14^{\prime \prime} .4$ & $-7^{\circ} 43^{\mathrm{m}} 35^{\prime \prime}$ & $20.544 \pm 0.008$ & $0.979 \pm 0.011$ & 0.982 & $582 \pm 35$ & 19 & $\sqrt{ }$ \\
\hline 13 & HST18 & $10^{\mathrm{h}} 05^{\mathrm{m}} 15^{\prime \prime} .2$ & $-7^{\circ} 43^{\mathrm{m}} 34^{\prime \prime}$ & $20.970 \pm 0.010$ & $0.989 \pm 0.013$ & 1.015 & $546 \pm 28$ & 12 & $\sqrt{ }$ \\
\hline $14 \mathrm{a}$ & HST27 & $10^{\mathrm{h}} 05^{\mathrm{m}} 16^{\prime \prime} 6$ & $-7^{\circ} 43^{\mathrm{m}} 29^{\prime \prime}$ & $21.266 \pm 0.011$ & $1.105 \pm 0.015$ & 1.117 & $321 \pm 25$ & 11 & $\sqrt{ }$ \\
\hline $14 \mathrm{~b}$ & HST12 & $10^{\mathrm{h}} 05^{\mathrm{m}} 17^{\prime \prime} .2$ & $-7^{\circ} 43^{\mathrm{m}} 20^{\prime \prime}$ & $20.522 \pm 0.007$ & $1.159 \pm 0.009$ & 1.138 & $696 \pm 15$ & 23 & $\sqrt{ }$ \\
\hline 15 & HST17 & $10^{\mathrm{h}} 05^{\mathrm{m}} 16^{\prime \prime} 8$ & $-7^{\circ} 43^{\mathrm{m}} 09^{\prime \prime}$ & $20.946 \pm 0.010$ & $0.978 \pm 0.013$ & 1.021 & $739 \pm 23$ & 16 & $\sqrt{ }$ \\
\hline 16 & HST32 & $10^{\mathrm{h}} 05^{\mathrm{m}} 18^{\prime \prime} 0$ & $-7^{\circ} 43^{\mathrm{m}} 15^{\prime \prime}$ & $21.467 \pm 0.012$ & $0.913 \pm 0.016$ & 0.878 & $599 \pm 54$ & 12 & $\sqrt{ }$ \\
\hline 17 & HST09 & $10^{\mathrm{h}} 05^{\mathrm{m}} 17^{\prime \prime} .2$ & $-7^{\circ} 42^{\mathrm{m}} 49^{\prime \prime}$ & $20.393 \pm 0.007$ & $1.147 \pm 0.010$ & 1.167 & $813 \pm 17$ & 23 & $\sqrt{ }$ \\
\hline 18 & HST31 & $10^{\mathrm{h}} 05^{\mathrm{m}} 18^{\prime \prime} .4$ & $-7^{\circ} 42^{\mathrm{m}} 45^{\prime \prime}$ & $21.392 \pm 0.012$ & $1.190 \pm 0.016$ & 1.236 & $210 \pm 23$ & 9 & $\sqrt{ }$ \\
\hline 19 & HST57 & $10^{\mathrm{h}} 05^{\mathrm{m}} 17^{\prime \prime} .3$ & $-7^{\circ} 42^{\mathrm{m}} 08^{\prime \prime}$ & $21.921 \pm 0.018$ & $0.891 \pm 0.028$ & 0.983 & $736 \pm 111$ & 5 & $\sqrt{ }$ \\
\hline 20 & HST46 & $10^{\mathrm{h}} 05^{\mathrm{m}} 17^{\prime \prime} .6$ & $-7^{\circ} 41^{\mathrm{m}} 57^{\prime \prime}$ & $21.802 \pm 0.015$ & $0.919 \pm 0.023$ & 0.946 & $661 \pm 55$ & 7 & $\sqrt{ }$ \\
\hline 21 & - & $10^{\mathrm{h}} 05^{\mathrm{m}} 20^{\prime} 1$ & $-7^{\circ} 42^{\mathrm{m}} 16^{\prime \prime}$ & - & - & 0.971 & $846 \pm 42$ & 12 & $\sqrt{ }$ \\
\hline 22 & - & $10^{\mathrm{h}} 05^{\mathrm{m}} 19^{\prime} 0$ & $-7^{\circ} 41^{\mathrm{m}} 47^{\prime \prime}$ & - & - & 0.957 & $670 \pm 41$ & 7 & $\sqrt{ }$ \\
\hline 23 & WHT15 & $10^{\mathrm{h}} 05^{\mathrm{m}} 20^{\prime} \cdot 2$ & $-7^{\circ} 41^{\mathrm{m}} 45^{\prime \prime}$ & - & - & 1.140 & $682 \pm 15$ & 34 & $\sqrt{ }$ \\
\hline 24 & WHT16 & $10^{\mathrm{h}} 05^{\mathrm{m}} 21^{\prime \prime} \cdot 2$ & $-7^{\circ} 41^{m} 38^{\prime \prime}$ & - & - & 0.992 & $1008 \pm 18$ & 42 & $\sqrt{ }$ \\
\hline $25 \mathrm{a}$ & - & $10^{\mathrm{h}} 05^{\mathrm{m}} 23^{\prime \prime} .4$ & $-7^{\circ} 41^{\mathrm{m}} 59^{\prime \prime}$ & - & - & 0.832 & $202 \pm 42$ & 19 & $\sqrt{ }$ \\
\hline $25 b$ & - & $10^{\mathrm{h}} 05^{\mathrm{m}} 23^{\prime \prime} \cdot 7$ & $-7^{\circ} 41^{\mathrm{m}} 55^{\prime \prime}$ & - & - & 0.890 & $721 \pm 30$ & 46 & $\sqrt{ }$ \\
\hline $26 \mathrm{a}$ & - & $10^{\mathrm{h}} 05^{\mathrm{m}} 22^{\prime \prime} \cdot 7$ & $-7^{\circ} 41^{\mathrm{m}} 13^{\prime \prime}$ & - & - & 1.068 & $803 \pm 24$ & 10 & $\sqrt{ }$ \\
\hline $26 b$ & WHT17 & $10^{\mathrm{h}} 05^{\mathrm{m}} 22^{\prime \prime} 9$ & $-7^{\circ} 41^{\mathrm{m}} 07^{\prime \prime}$ & - & - & 0.934 & $769 \pm 25$ & 19 & $\sqrt{ }$ \\
\hline
\end{tabular}

${ }^{a}$ Background galaxy where [OII] emission is visible.

${ }^{b}$ HSTxx denotes globular clusters selected from the HST study of Kundu \& Whitmore (1998), WHTxx are objects from the investigation of Kavelaars (1998) while "-." indicates objects selected from our own VLT-FORS2 image in order to fill the mask.

${ }^{c}$ Pseudo $(V-I)$ colour derived from the globular cluster spectra. The mean error is 0.037 mag. See Sect. 3 for details.

The seventh column shows pseudo $(V-I)$ colours derived from our spectroscopy (see Sect. 3 for details). Columns eight and nine list the radial velocity and the mean $S / N$ per pixel respectively. Finally, column ten indicates whether the GC candidate is a spectroscopically confirmed member of the NGC 3115 system (see next paragraph).

The radial velocity of NGC 3115 is listed as $720 \pm 5 \mathrm{~km} \mathrm{~s}^{-1}$ by Smith et al. (2000). Kavelaars (1998) find a mean velocity of $620 \mathrm{~km} \mathrm{~s}^{-1}$ and a velocity dispersion of $\sigma=177 \mathrm{~km} \mathrm{~s}^{-1}$ from low resolution spectroscopic observations of 22 globular clusters. We consider all objects with radial velocities between $200 \mathrm{~km} \mathrm{~s}^{-1}$ and $1300 \mathrm{~km} \mathrm{~s}^{-1}$ as members of the NGC 3115 system. Object 6 is a background galaxy with a redshift of $z=0.39$, while object 4 is likely to be a galactic star. In total 26 out of $28 \mathrm{GC}$ candidates (93\%) turned out to be objects which are dynamically associated with NGC 3115. This low contamination fraction is to be expected for a sample of candidates predominantly selected by morphological criteria from HST data.

In order to allow a proper line-strength analysis we restrict our sample to GCs with a mean $S / N \geq 12$ per pixel $^{1}$ yielding a final sample of 17 GCs in NGC 3115. Below this signal-tonoise cut the line-strength errors are too large to allow a proper age-metallicity analysis. The mean $S / N$ of our final sample is 27 per pixel (ranging from 12 to 61 ). The GCs have a mean

1 The $S / N$ is determined as an average over the observed wavelength range. The minimum $S / N$ per resolution element is $\approx 25$. 


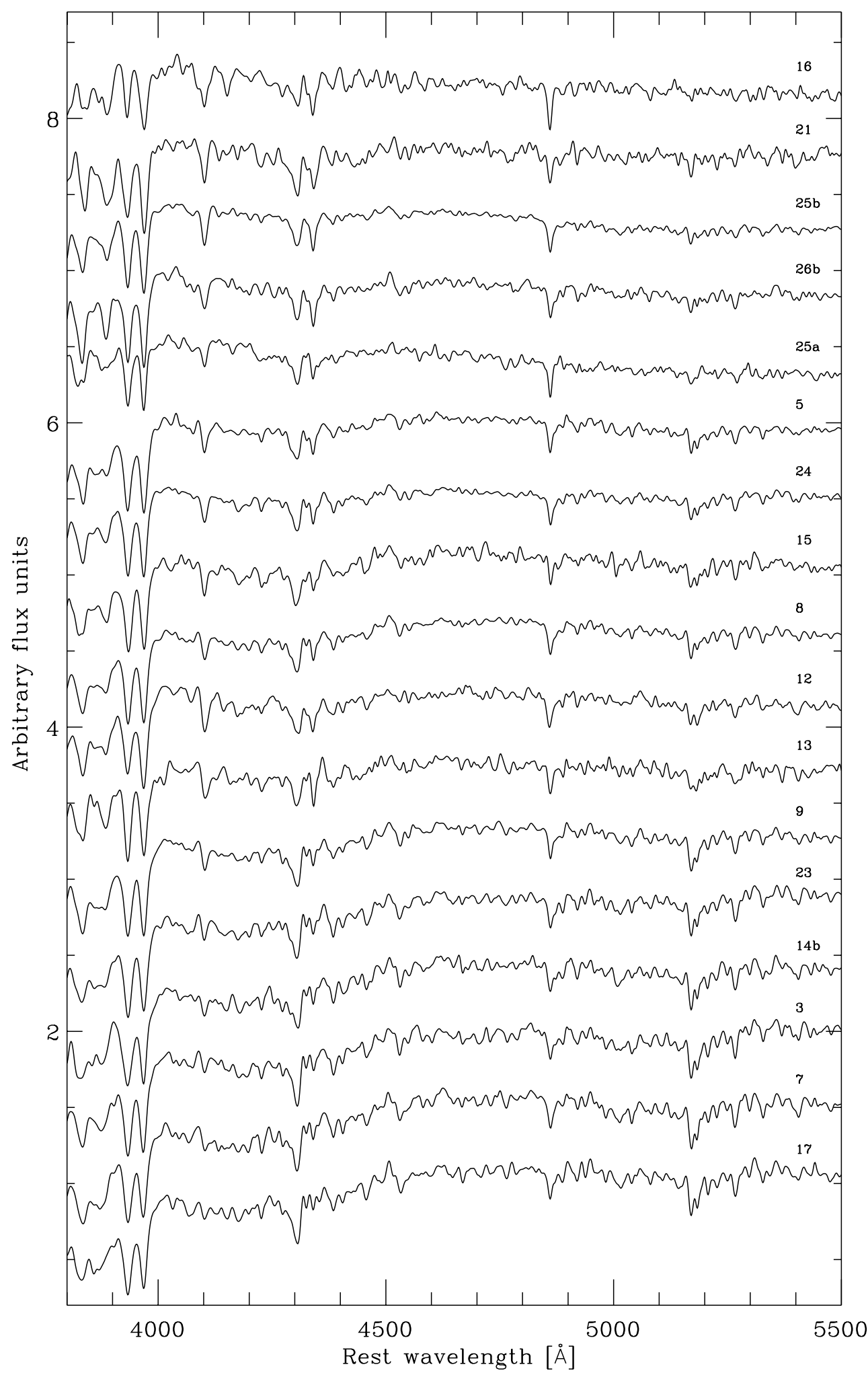

Fig. 1. Rest-frame spectra of globular clusters corrected to the Lick/IDS resolution and sorted by increasing strength of our mean metallicity indicator $[\mathrm{MgFe}]$ (from top to bottom, see also Sect. 4). Note the change in overall continuum shape (metal poorer globular clusters are bluer) and also the change in absorption strength of the Balmer lines ( $\mathrm{H} \delta$ at $4101 \AA, \mathrm{H} \gamma$ at $4340 \AA$ and $\mathrm{H} \beta$ at $4861 \AA$ ). The slitlet ID for each spectrum is indicated at the right hand side of the plot (see also Table 1).

velocity of $600 \mathrm{~km} \mathrm{~s}^{-1}$ ( $1 \sigma$ scatter: $\left.215 \mathrm{~km} \mathrm{~s}^{-1}\right)$. The spectra of these 17 GCs broadened to the Lick/IDS resolution are shown in Fig. 1.
We note that the globular cluster 14a (HST27, $S / N=11$ ) shows clear $[\mathrm{OIII}] \lambda 5007$ emission which is however redshifted by $\approx 190 \mathrm{~km} \mathrm{~s}^{-1}$ with respect to the recession velocity of the 
globular cluster itself. Therefore the emission cannot be associated with the globular cluster itself, although the spatial extent of the emission is consistent with the size of the GC on the sky.

Lick indices (for index definitions see Worthey \& Ottaviani 1997; Trager et al. 1998) were measured from the resolution and continuum corrected spectra covering a common observed wavelength range of 3670-5500 A. Uncertainties in the indices were derived by Monte-Carlo simulations which take into account the photon noise, read-out noise of the CCD and the errors in the velocity determination. The Lick system is based on non-flux calibrated spectra, so one expects small offsets (e.g., Kuntschner 2000) in the zero-point of the line indices. Since no stars in common with the Lick stellar library were observed during our observing run we use the offsets established by Vazdekis (1999) for a large flux-calibrated sample of stars ("Jones library") to transform our index measurements onto the Lick system. Since typical metallicities for GCs are between $-2.0<[\mathrm{Fe} / \mathrm{H}]<0.0$, we calculated the offset by averaging the values for $[\mathrm{Fe} / \mathrm{H}]=-0.7$ and -0.4 given by Vazdekis (1999 see his Table 2). Note, that some indices (e.g., $\mathrm{Mg}_{2}$ ) show strong evidence for a metallicity dependent offset, which can introduce systematic offsets as a function of line-strength. Here in this paper we use mainly indices where the former problem is only a second order effect. The measured index values and their associated errors are listed in Tables A.1 and A.2 in the Appendix.

The positions of the GCs with respect to the parent galaxy are shown in Fig. 2. Due to the optimisation of the target efficiency and the observational constraints of the FORS2 instrument the GCs lie in a narrow band $\sim 25^{\prime \prime}$ eastwards of the galaxy centre parallel to the major axis of NGC 3115 . Both the red and blue sub-samples were evenly distributed across the CCD.

In Fig. 3 we assess the kinematics of all globular clusters which have recession velocities consistent with being members of the NGC 3115 system. There is a strong signature of GC rotation along the major axis of the galaxy. We confirm here the weak signal of rotation for the red cluster population detected by Kavelaars (1998). In our sample there is no clear difference between red and blue clusters, both show an equally strong signal of rotation.

Since our sample of GCs is dominated by clusters close to the location of the major axis (see Fig. 2) we note that the sample is probably biased to globular clusters associated with the disk-formation of NGC 3115.

\section{Colour distribution}

9 out of 17 GCs for which we have good quality spectra and are kinematically associated with NGC 3115 are within the HST/WFPC2 image. In order to assign colours to the remaining $8 \mathrm{GCs}$ we derived an empirical relationship between a "pseudo-colour" measured from our flux-calibrated spectra and the HST $(V-I)$ colours. Of course our limited wavelength range does not allow us to measure a real $(V-I)$ colour but one can get a rough idea on the overall spectral shape. On the spectra we measured the mean counts in two regions (3900$4600 \AA$ and $4800-5500 \AA$ ) and plotted the difference versus

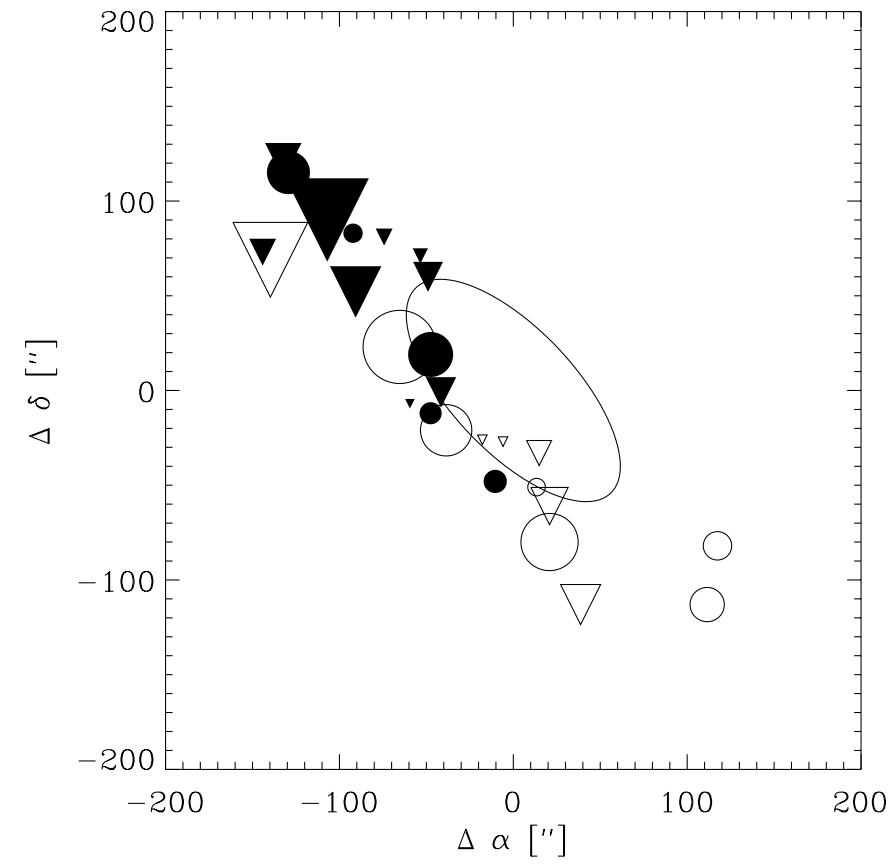

Fig. 2. Positions of spectroscopically confirmed globular clusters with respect to the centre of NGC 3115 (triangles represent blue clusters and circles red clusters, see Sect. 3). Approaching and receding globular clusters are indicated by open symbols and filled symbols respectively, while the symbol size indicates the extent of the velocity difference with respect to the mean velocity of the whole sample. The ellipse encloses half of the integrated light and indicates the position and orientation of the main galaxy itself (data from Michard \& Marchal 1994). North is up and east to the left.

the HST $(V-I)$ colour (see Fig. 4). This procedure yields a linear relationship with a mean scatter of $0.037 \mathrm{mag}$ in derived $(V-I)$ colour. Note that here we have used all available spectra of GC candidates with measured HST colours (see Table 1), specifically including the ones with an $S / N<12$ per pixel. The derived $(V-I)$ colours are listed in Table 1 in column seven where we have assigned a mean error of $0.037 \mathrm{mag}$.

The colour distribution of our sample of 17 GCs is shown in Fig. 5 together with the full HST sample by Kundu \& Whitmore (1998). The HST sample shows a clear bimodal structure with peaks at $(V-I)=0.96$ and $(V-I)=1.17$. Taking the mean colour of the HST sample $((V-I)=1.06)$ as the dividing line between the red and blue clusters, our spectroscopic sample features eleven blue clusters and six red ones while spanning the range $(V-I)=0.87-1.18$.

\section{Treatment of abundance ratios}

Theory of chemical enrichment predicts that stellar populations created in a short burst of star formation show elevated magnesium-to-iron abundance ratios while extended periods of star formation lead to roughly solar abundance ratios (e.g., Tinsley 1979; Worthey et al. 1992; Worthey 1998; Thomas et al. 1999). This is generally explained by either a delay in the production of $\mathrm{Fe}$-peak elements with respect to $\alpha$-elements due to the different timescales of SN Ia and SN II, or by a starburst with an initial mass function skewed to massive stars. 


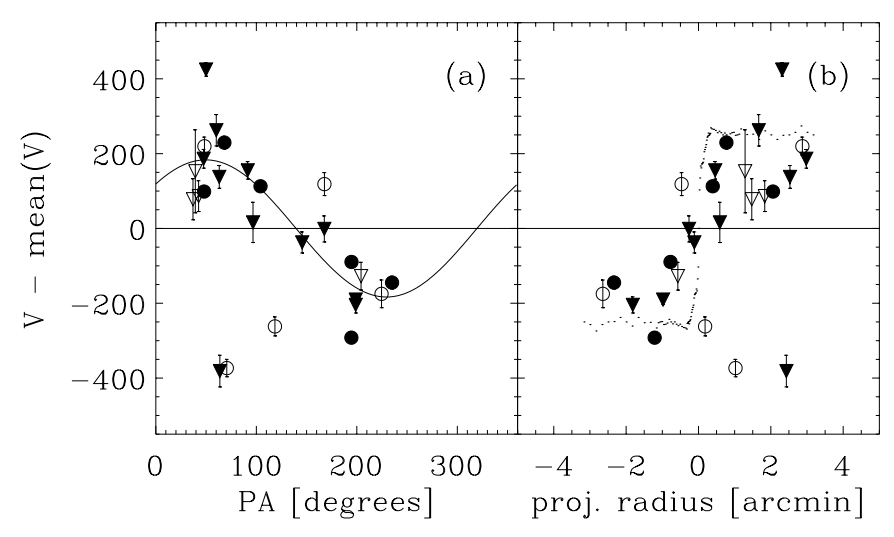

Fig. 3. Assessment of rotation for NGC 3115 globular clusters. The triangles and circles represent blue and red clusters, respectively. Filled symbols indicate the high $S / N$ sample of 17 globular clusters for which we present a line-strength analysis in this paper. Panel a): The vertical axis shows the radial velocity of the globular clusters relative to the mean velocity of the sample. The horizontal axis is the position angle between a globular cluster and the galaxy centre, where a PA of 0 degrees represents north and positive is east of north. The sinusoid is a simple least-squares fit to the full sample with one iteration to reject outliers. Panel b): radial velocity is plotted against the projected major axis radius. Positive numbers are towards north-east. The small dots represent the stellar rotation curve of NGC 3115 along the major axis (Capaccioli et al. 1993).

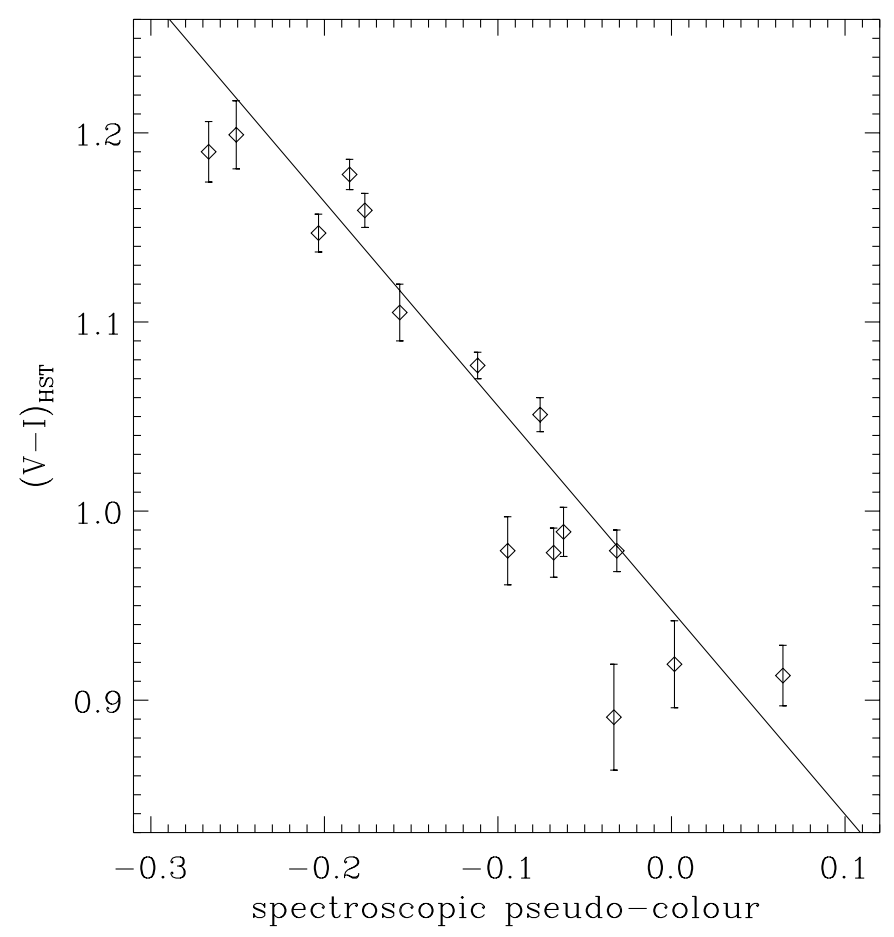

Fig. 4. The pseudo colour derived from the spectra versus $(V-I)_{\mathrm{HST}}$. The linear fit corresponds to $(V-I)_{\mathrm{HST}}=-1.08( \pm 0.04) \times$ $($ spec colour $)+0.95( \pm 0.01)$. For details see Sect. 3 .

For example, many observations of bright elliptical galaxies in clusters show indeed elevated magnesium-to-iron ratios indicating a rapid star formation (e.g., Peletier 1989; Worthey et al. 1992; Davies et al. 1993; Kuntschner 2000).

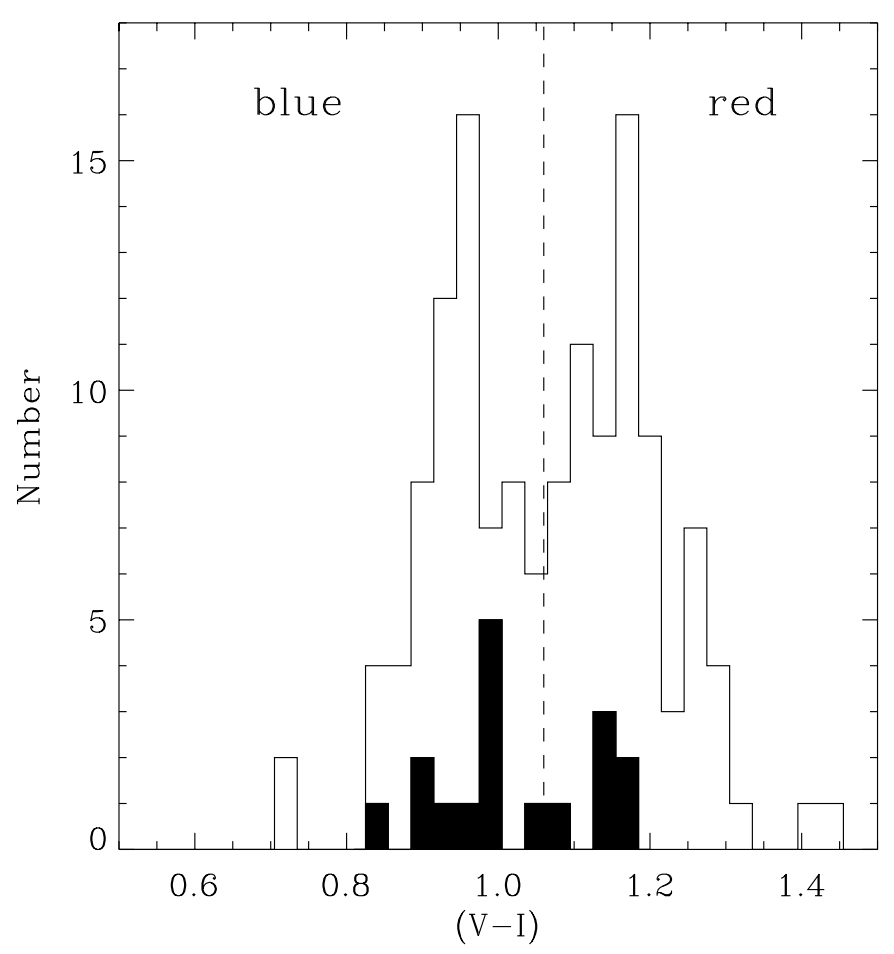

Fig. 5. Histogram of $(V-I)$ colours of the cluster candidates from Kundu \& Whitmore (1998). Overplotted as a filled histogram is our spectroscopic sample of 17 confirmed globular clusters in NGC 3115. The vertical dashed line indicates the dividing line between red and blue clusters at $(V-I)=1.06$.

GCs represent simple stellar populations (hereafter SSP, i.e., a unique age and chemical composition) since all the stars of one GC are thought to be created in a single star formation event. But it is not known a priori whether the stars of a given $\mathrm{GC}$ are formed out of $\mathrm{Fe}$-deficient gas clouds, which have been only enriched by SN II producing little Fe, or out of an already well mixed interstellar medium harbouring the products from both SN Ia (main producer of Fe) and SN II. For GC systems with a bimodal colour distribution like in NGC 3115, all scenarios that have been proposed to explain the origin of the red metal-rich GCs start from the principle that the red population is formed in a separate star formation event (e.g., Ashman $\&$ Zepf 1992; Forbes et al. 1997). In a naive star-formation scenario, where the red clusters form from the well-mixed interstellar medium they should show solar abundance ratios.

Most of the currently available stellar population models which can be used to investigate the abundance ratios of extragalactic objects are based on stellar libraries from our own Galaxy. This has the disadvantage that, particularly at subsolar metallicities, galactic disk stars show super-solar abundance ratios for many $\alpha$-elements (Edvardsson et al. 1993; McWilliam 1997). Therefore, without correction, the model predictions will be biased towards super-solar abundance ratios (e.g., Borges et al. 1995; Kuntschner 2000; Thomas et al. 2002a).

Thomas et al. (2002a) (see also Thomas et al. 2002b) provide new models which take the stellar library biases into account and can predict line-strengths for solar abundance as well 


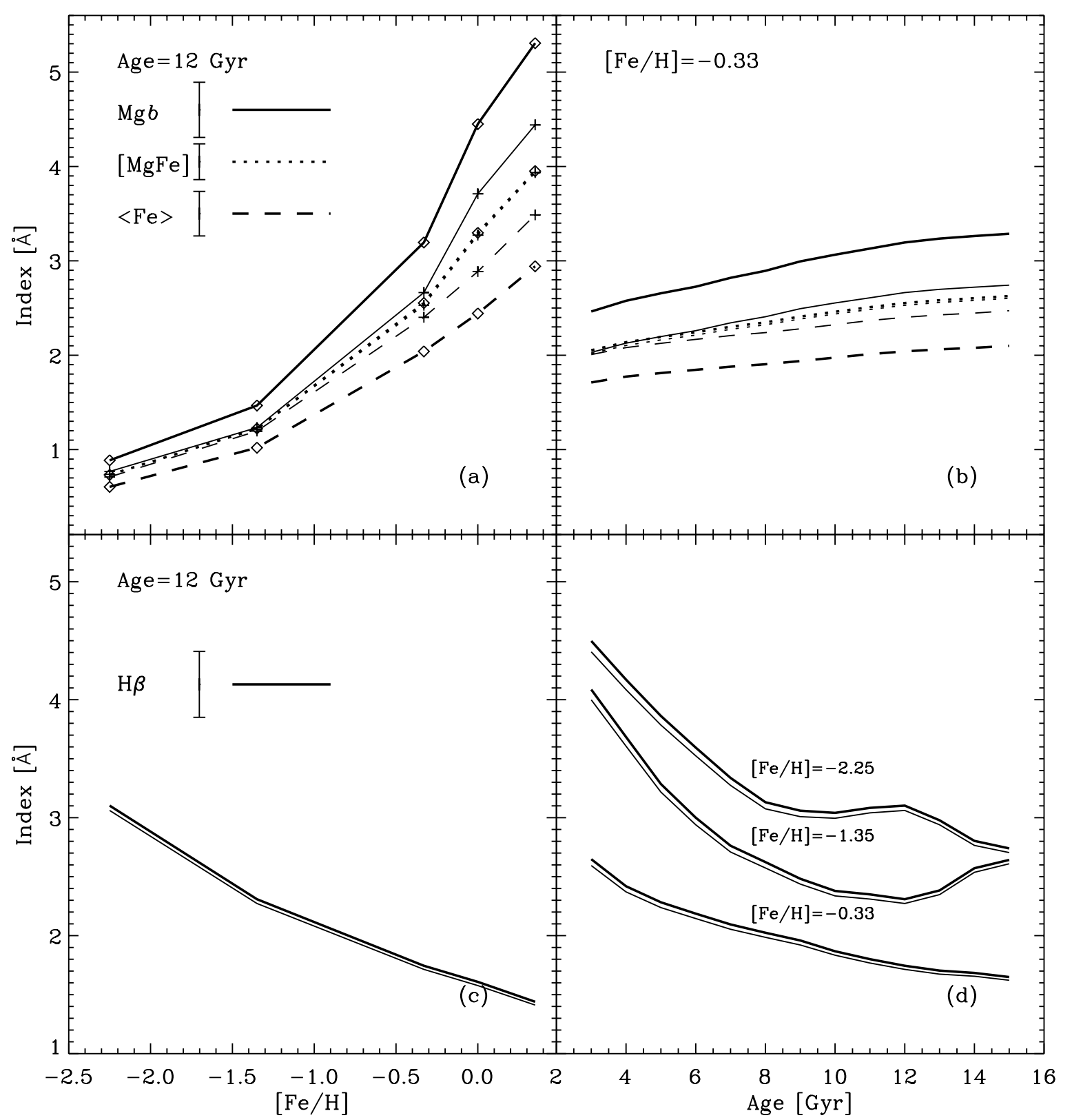

Fig. 6. Dependence of $\mathrm{Mg} b,[\mathrm{MgFe}],\langle\mathrm{Fe}\rangle$ and $\mathrm{H} \beta$ on $[\mathrm{Fe} / \mathrm{H}]$ and age for models with different $[\mathrm{Mg} / \mathrm{Fe}]$ as predicted by Thomas et al. (2002a). The thick lines and open diamonds stand for $[\mathrm{Mg} / \mathrm{Fe}]=+0.3$ whereas the thin lines and plus symbols represent $[\mathrm{Mg} / \mathrm{Fe}]=0.0$. The respective line-style and average measurement error for the different indices is given on the left hand side of panels a) and c). For the [MgFe] index the two model lines (for $[\mathrm{Mg} / \mathrm{Fe}]=0.0$ and 0.3 ) overlap almost completely and therefore the index is almost independent of the Mg to Fe abundance ratios.

as non-solar abundance ratios over a large metallicity range $(-2.25 \leq[\mathrm{Fe} / \mathrm{H}] \leq 0.35)$. Since our paper is among the first that make use of these new models for studying GC spectra, we first explore systematically how the three parameters age, metallicity and abundance ratio $([\mathrm{Mg} / \mathrm{Fe}])$ affect the absorption line-strengths of SSPs. For this purpose we plot in Fig. 6 the model predictions for the line-strengths of the often used metallicity indicators $\mathrm{Mg} b,\langle\mathrm{Fe}\rangle^{2}$ and $[\mathrm{MgFe}]^{3}$ and the age sensitive Balmer line $\mathrm{H} \beta$ as a function of metallicity, age and $[\mathrm{Mg} / \mathrm{Fe}]$.

${ }^{2}\langle\mathrm{Fe}\rangle=(\mathrm{Fe} 5270+\mathrm{Fe} 5335) / 2($ González 1993).

$3[\mathrm{MgFe}]=\sqrt{\mathrm{Mg} b \times(\mathrm{Fe} 5270+\mathrm{Fe} 5335) / 2}$ (González 1993).
The effects of non-solar abundance ratios (thick lines) with respect to solar ratio model predictions (thin lines) can be clearly seen for the metallicity sensitive indices $\mathrm{Mg} b$ and $\langle\mathrm{Fe}\rangle$. At a given age and all metallicities (Fig. 6a), the $\mathrm{Mg} b$ index is predicted to be stronger for super-solar $[\mathrm{Mg} / \mathrm{Fe}]$. The difference between solar ratio and non-solar ratio models increases with increasing metallicity. This behaviour is reversed for the $\langle\mathrm{Fe}\rangle$ index (see also Trager et al. 2000). A similar effect can be observed when we fix $[\mathrm{Fe} / \mathrm{H}]$ but vary age (Fig. $6 \mathrm{~b}$ ). Remarkably, the $[\mathrm{MgFe}]$ index, the geometric mean of the $\mathrm{Mg} b$ and $\langle\mathrm{Fe}\rangle$ indices, does not show any significant dependence on abundance ratios, at least not within the current framework of the models. 


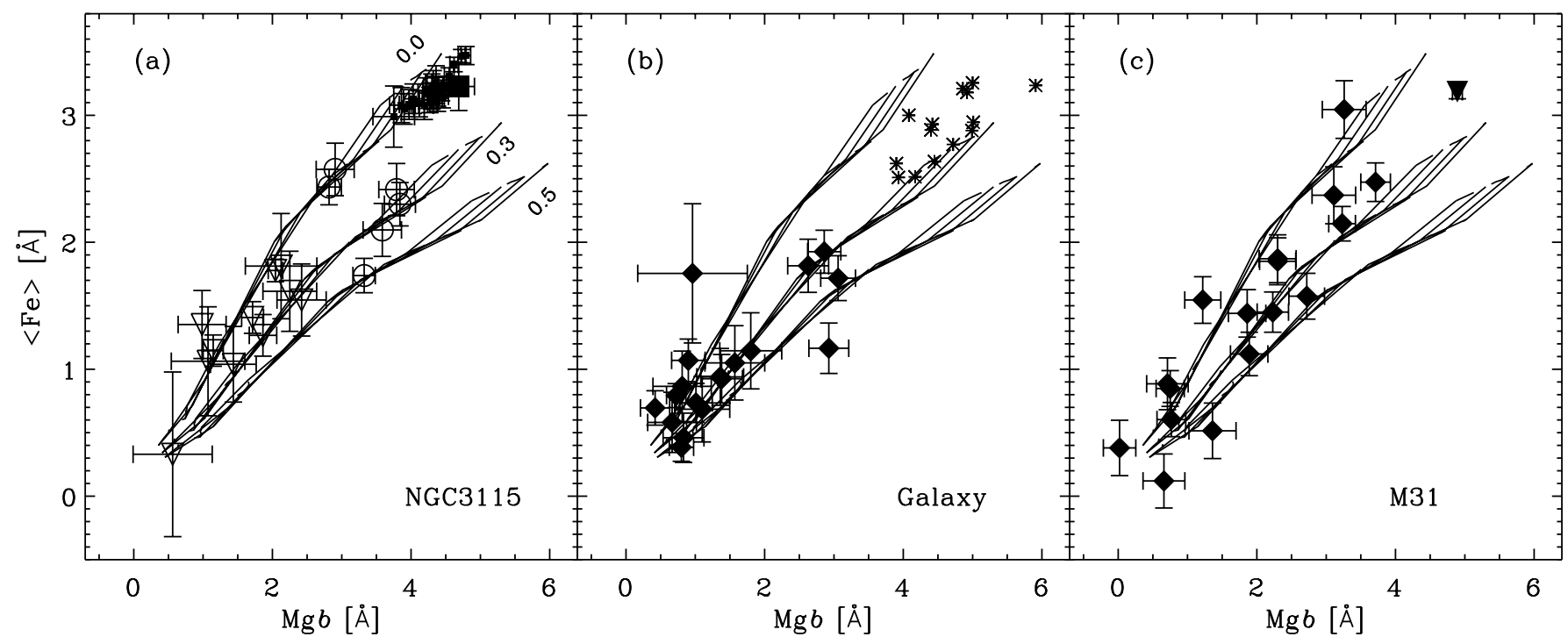

Fig. 7. Probing the $[\mathrm{Mg} / \mathrm{Fe}]$ ratios of globular clusters in a $\mathrm{Mg} b$ vs. $\langle\mathrm{Fe}\rangle$ diagram. Panel a): Our sample of globular clusters in NGC 3115 is shown as open triangles (blue clusters) and open circles (red clusters). The large filled square represents the centre of NGC 3115 taken from Trager et al. (1998) and the small filled squares represent the data of Fisher et al. (1996) which cover radii up to 40" along the major axis. Panel b): Milky Way globular clusters observed with the Lick/IDS instrumentation. Overplotted as stars are early-type galaxies in the Fornax cluster with central velocity dispersion $\sigma \geq 75 \mathrm{~km} \mathrm{~s}^{-1}$ (Kuntschner 2000). Panel c): Globular clusters in M31 observed with the Lick/IDS instrumentation. The filled triangle represents the centre of M31 taken from Trager et al. (1998). Overplotted in all panels are models by Thomas et al. (2002a) with abundance ratios of $[\mathrm{Mg} / \mathrm{Fe}]=0.0 ., 0.3,0.5$ as indicated in the left panel. The models span a range in age $(3-12 \mathrm{Gyr})$ and metallicity $([\mathrm{Fe} / \mathrm{H}]=-2.35$ to +0.3$)$.

This has the unique advantage that $[\mathrm{MgFe}]$ can be used as an empirical mean metallicity indicator with negligible dependence on the abundance ratios (see also Kuntschner et al. 2001). Similarly, $\mathrm{H} \beta$ is hardly affected by $[\mathrm{Mg} / \mathrm{Fe}]$ with a small increase in line-strength for larger $[\mathrm{Mg} / \mathrm{Fe}]$ ratios (Figs. $6 \mathrm{c}$ and $\mathrm{d}$ ).

However, the $\mathrm{H} \beta$ index shows a noteworthy complication at low metallicities $([\mathrm{Fe} / \mathrm{H}] \leq-1.35)$ and ages $>8$ Gyrs. Here the models predict a non-monotonic decrease of the $\mathrm{H} \beta$ index with increasing age (see Fig. $6 \mathrm{~d}$, models for $[\mathrm{Fe} / \mathrm{H}]=-2.25$ and -1.35 ). Therefore, in this age and metallicity range a given measurement of $\mathrm{H} \beta$ and $[\mathrm{MgFe}]$ indices does not correspond to one unique age but can in fact be consistent with a range of ages. The ambiguity arises between a genuine measurement of the turn-off temperature by $\mathrm{H} \beta$ and the appearance of blue horizontal branch stars in old, metal poor stellar systems which will start to increase the $\mathrm{H} \beta$ index, mimicking a younger age (Lee et al. 2000; Maraston \& Thomas 2000; Beasley et al. 2002b). The overall result is a "crossing" of iso-age lines at ages larger than 8 Gyr and low metallicities. In Sect. 5.2 where we will determine our best age estimates, the above effect will be taken into account.

\section{Results}

We first present our results on the abundance ratios of $\mathrm{Mg}$ to $\mathrm{Fe}$ of GCs in NGC 3115 and compare these with the Lick/IDS observations (Trager et al. 1998) of GCs in our own galaxy and M31. In Sect. 5.2 we present our best estimates of the ages and metallicities of NGC 3115 GCs, while we compare photometric and spectroscopic metallicity estimates in Sect. 5.3.

\subsection{Abundance ratios}

In Fig. 7, we show a diagram of $\mathrm{Mg} b$ vs. $\langle\mathrm{Fe}\rangle$ index. We plot our sample of GCs separated by $(V-I)$ colour (see Fig. 5) where red and blue clusters are shown as open circles and open triangles respectively. Overplotted are the model predictions by Thomas et al. (2002a) for $[\mathrm{Mg} / \mathrm{Fe}]=0.0,0.3,0.5$, age $=$ $3,5,8,12 \mathrm{Gyr}$ and $[\mathrm{Fe} / \mathrm{H}]=-2.25,-1.35,-0.33,0.0,+0.35$. In this diagram the effects of age and metallicity are almost completely degenerate and the sensitivity towards abundance ratios is maximised. The models predict a significant change in line-strength for varying abundance ratios at large metallicities/ages (i.e. large $\mathrm{Mg} b \&\langle\mathrm{Fe}\rangle$ line-strength) while at low metallicities the dependence is smaller. Therefore, at a given error in line-strength, abundance ratios are determined more accurately at larger metallicities/ages.

We find that for GCs in NGC 3115 the abundance ratios vary from roughly solar to about $[\mathrm{Mg} / \mathrm{Fe}] \simeq+0.3$ (with a maximum of $[\mathrm{Mg} / \mathrm{Fe}] \simeq+0.5$, see Fig. $7 \mathrm{a}$ ). In our small sample there is no evidence for a trend with colour. In particular, there are GCs with solar and super-solar abundance ratios in both the blue and red GC populations. There is even some weak evidence for a bimodal abundance ratio distribution with peaks at $[\mathrm{Mg} / \mathrm{Fe}] \approx 0.0$ and $\approx 0.3$ (see Fig. 8 ). This tentative evidence needs to be confirmed with a larger sample of GCs.

The measurements for the centre of NGC 3115 itself (large filled square, data from Trager et al. 1998) and the radial gradient (small filled squares, data from Fisher et al. 1996) along the major axis up to $40^{\prime \prime}$ is compatible with a model of $[\mathrm{Mg} / \mathrm{Fe}] \approx 0.0$ at high metallicity/age. In Figs. $7 \mathrm{~b}$ and $\mathrm{c}$ we plot the Lick/IDS observations of GCs in the Milky Way and M31 


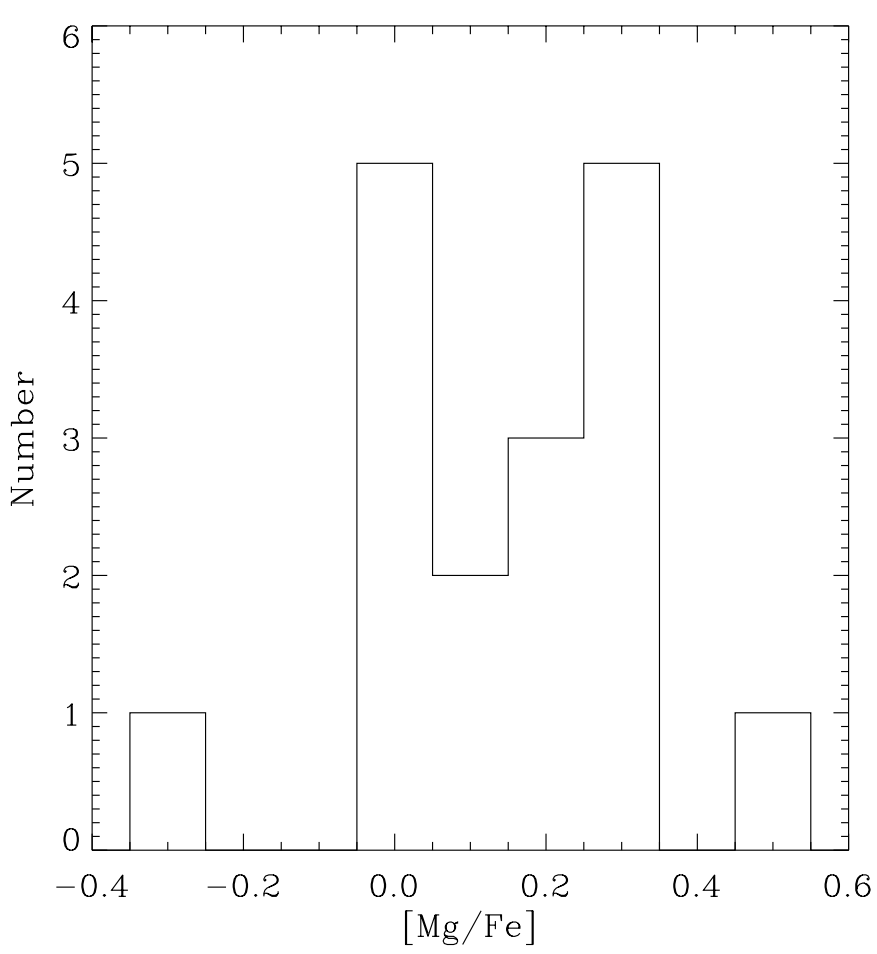

Fig. 8. Histogram of abundance ratios $[\mathrm{Mg} / \mathrm{Fe}]$ for NGC 3115 globular clusters. The abundance ratios were determined from Fig. 7a.

respectively (data from Trager et al. 1998). While virtually all MW GCs are consistent with $[\mathrm{Mg} / \mathrm{Fe}] \simeq+0.3$, similar to large elliptical galaxies, we find a range in $[\mathrm{Mg} / \mathrm{Fe}]$ for the GCs in $\mathrm{M} 31$. The overall distribution of the $[\mathrm{Mg} / \mathrm{Fe}]$ ratios for $\mathrm{GCs}$ in M 31 is similar to the one we find in NGC 3115. The average value of $[\mathrm{Mg} / \mathrm{Fe}]=+0.3$ we find for the MW GCs compares well with high resolution studies of individual stars in MW GCs (e.g., Lee \& Carney 2002).

GCs with super-solar $[\mathrm{Mg} / \mathrm{Fe}]$ ratios were previously found in other nearby galaxies but without our quantitative accuracy. For example, Forbes et al. (2001) attribute super-solar abundance ratios to 4 out of $10 \mathrm{GCs}$ in NGC 1399. Using Mg and $\mathrm{TiO}$ features, Larsen et al. (2002) find a mean $[\alpha / \mathrm{Fe}]$ of +0.4 for both metal-poor and metal-rich GCs in the Sombrero galaxy.

\subsection{Age and metallicity}

In the previous section we were able to determine the abundance ratios of GCs without knowing the age and metallicity since the latter parameters are almost completely degenerate in a $\mathrm{Mg} b$ vs. $\langle\mathrm{Fe}\rangle$ diagnostic diagram. However, our earlier discussion of the model systematics shows that we need to take the abundance ratios into account in order to estimate the age and metallicity of the GCs (see also e.g., Trager et al. 2000; Kuntschner et al. 2001).

Principle age sensitive lines within our observed wavelength range are the Balmer lines $\mathrm{H} \beta, \mathrm{H} \gamma$, and $\mathrm{H} \delta$. For $\mathrm{H} \gamma$ and $\mathrm{H} \delta$, the dependence on $\alpha$-element to Fe ratio is yet unknown. $\mathrm{H} \beta$ is only marginally sensitive to abundance ratio variations, at least in comparison to our average observational error. To further minimise the influence of abundance ratios, we employ as metallicity indicator [ $\mathrm{MgFe}]$, that also shows no significant $[\mathrm{Mg} / \mathrm{Fe}]$ dependence (see Fig. 6). Within the accuracy of our data sample, an $\mathrm{H} \beta$ vs. $[\mathrm{MgFe}]$ diagram can therefore be used to estimate the ages and metallicities of our NGC 3115 GCs without being significantly affected by abundance ratios.

In Fig. 9 we show diagrams of $[\mathrm{MgFe}]$ versus the three Balmer lines for our sample of NGC 3115 GCs (left panels) and the respective data for GCs in the Milky Way and M 31 from the Lick/IDS observations (middle and right panels; the index values for $\mathrm{H} \beta, \mathrm{Mg} b$ and $\langle\mathrm{Fe}\rangle$ were taken from Trager et al. (1998); the higher order Balmer lines of the Lick/IDS observations are presented in Tables A.3 and A.4 in the Appendix). Overplotted in Fig. 9 are solar-abundance ratio models by Thomas et al. (2002a) and Maraston (2002, in preparation) for metallicities $[\mathrm{Fe} / \mathrm{H}]=-2.25,-1.35,-0.33,0.00,0.35$ (dashed lines, left to right) and ages 3, 5, 8 and $12 \mathrm{Gyr}$ (solid lines, top to bottom).

We first caution that a direct comparison between models and our data to derive absolute ages and metallicities can be dangerous due to possible systematic calibration errors. However, we estimate that systematic observational errors are smaller than $0.1 \AA$ for the indices shown here and emphasize that relative comparisons within one sample will be significant.

The observed $\mathrm{H} \beta$ values for NGC 3115 GCs show a large spread with respect to the model predictions. However, the data points which are well below the model predictions are the ones with the largest errors. Most of the well determined data points are close to the region of a 12 Gyr SSP model. Our $[\mathrm{MgFe}]$ measurements show that there is a clear distinction in metallicity between blue (open triangles) and red clusters (open circles), with the red clusters being more metal rich (a more detailed analysis of the metallicity distribution is presented in Sect. 5.3).

Since only about half of our data points have small enough error bars to be useful for an individual age/metallicity evaluation we also calculate the error weighted mean of the blue and red clusters, respectively. These average values (filled symbols in Fig. 9, left panels) give for the metal poor (blue) population an age of $12.0\left({ }_{-2.0}^{+1.5}\right) \mathrm{Gyr}$ and $[\mathrm{Fe} / \mathrm{H}]=-1.05( \pm 0.09)$, while the metal rich (red) population has an estimated age of $10.8\left({ }_{-1.8}^{+1.7}\right) \mathrm{Gyr}$ and $[\mathrm{Fe} / \mathrm{H}]=-0.26( \pm 0.05)$. The errors on the age and metallicity are quoted as $1 \sigma$ errors on the mean values.

We note in Sect. 4 that for metallicities $[\mathrm{Fe} / \mathrm{H}] \leq-1.35$ and an age larger than $8 \mathrm{Gyr}$ the strength of the $\mathrm{H} \beta$ and $[\mathrm{MgFe}]$ indices is not uniquely connected to one age anymore. In fact there is a "crossing" of iso-age curves. For clarity we do not plot iso-age lines for ages greater than 12 Gyr in Fig. 9, but this effect has been taken into account when deriving the errors on our best age and metallicity estimates.

From the $\mathrm{H} \beta$ vs. [MgFe] diagram we conclude that within our observational errors the two populations of GCs in NGC 3115 have the same age of 11-12 Gyr (assuming the calibration of models and data is accurate). The observed indices for the integrated light in the centre of NGC 3115 (taken from and shown as filled square in Fig. 9 Trager et al. 1998) are consistent with a luminosity weighted age of $\approx 12 \mathrm{Gyr}$.

The Lick/IDS observations of MW GCs also show a significant number of objects below the model predictions. We note 

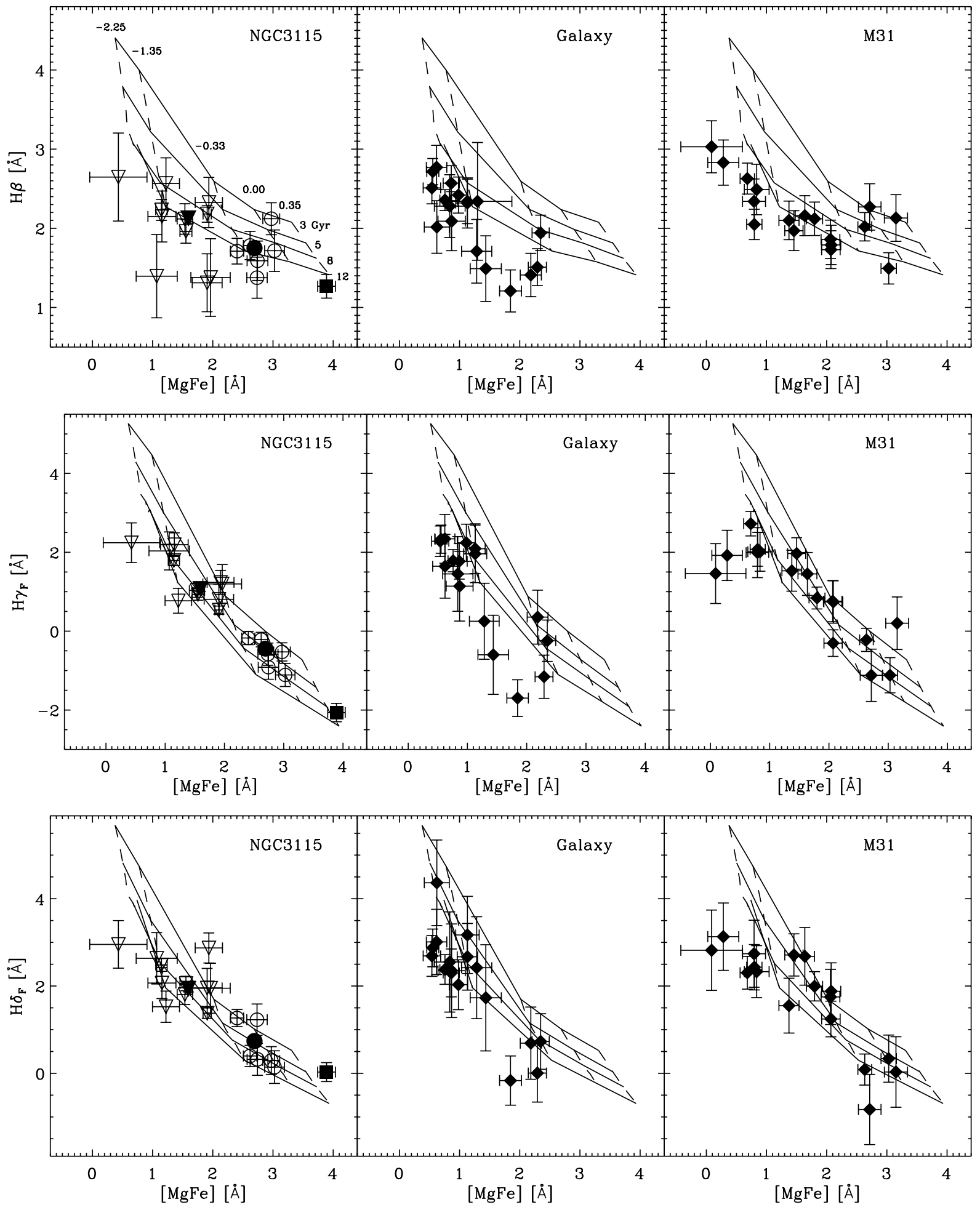

Fig. 9. Age and metallicity diagnostic diagrams using as metallicity indicator [MgFe] and as age indicator $\mathrm{H} \beta, \mathrm{H} \gamma_{\mathrm{F}}$, and $\mathrm{H} \delta_{\mathrm{F}}$. $\mathrm{H} \beta$ and $[\mathrm{MgFe}]$ are not significantly influenced by abundance ratios, for $\mathrm{H} \gamma_{\mathrm{F}}$, and $\mathrm{H} \delta_{\mathrm{F}}$ the behaviour is unknown. Our sample of globular clusters in NGC 3115 is shown in the left panels as open triangles (blue clusters) and open circles (red clusters). The error weighted means of the blue and red clusters are shown as filled symbols. The filled square in the left panels represents the centre of NGC 3115 Trager et al. (1998). The middle and right panels show the Lick/IDS data for Milky Way MW and M 31 globular clusters, respectively. Note, that for the MW globular cluster NGC 2158 there are no $\mathrm{H} \gamma_{\mathrm{F}}$ and $\mathrm{H} \delta_{\mathrm{F}}$ index measurements available. Overplotted are solar-abundance SSP models by Thomas et al. (2002a) and Maraston (2002, in preparation) for metallicities $[\mathrm{Fe} / \mathrm{H}]=-2.25,-1.35,-0.33,0.00,0.35$ (dashed lines, left to right) and ages $3,5,8$, and $12 \mathrm{Gyr}$ (solid lines, top to bottom). 
that there are no systematic observational offsets to be expected since the data was taken with the original Lick/IDS system. We speculate that observations of these GCs may be contaminated by fore/back-ground stars. New spectroscopic observations of MW GCs (Puzia et al. 2002a) support this hypothesis since the new observations do not show such low $\mathrm{H} \beta$ values. Consistent with recent age estimates from the resolved stellar populations of MW GCs (e.g., Rosenberg et al. 1999; Salaris $\&$ Weiss 2002) we do not find evidence for clusters younger than $\approx 8$ Gyr.

The Lick/IDS observations for M 31 GCs show a relatively small scatter close to a $12 \mathrm{Gyr}$ model prediction, with only three, metal-rich clusters showing evidence of a younger age. We note however, that for metallicities $[\mathrm{Fe} / \mathrm{H}]<-1.35$ the models seem to systematically over-estimate the $\mathrm{H} \beta$ absorption strength (or alternatively over-estimate the $[\mathrm{MgFe}]$ absorption strength).

In the next paragraphs we will present our measurements of the higher order Balmer lines $\mathrm{H} \gamma_{\mathrm{F}}$ and $\mathrm{H} \delta_{\mathrm{F}}$. We emphasize here that while these indices can be measured with a higher precision than $\mathrm{H} \beta$, it is currently unknown how these indices depend on abundance ratios. Furthermore the absolute calibration of these indices has not yet been investigated in as much detail as the $\mathrm{H} \beta$ index.

The distributions of $\mathrm{H} \gamma_{\mathrm{F}}$ and $\mathrm{H} \delta_{\mathrm{F}}$ vs $[\mathrm{MgFe}]$ are narrower compared to $\mathrm{H} \beta$ vs. [MgFe] and mostly encompassed by the model grid. The error weighted means for NGC 3115 GCs indicate an age of $\approx 7$ and $\approx 5$ Gyr for the blue and red clusters, respectively. These average ages are substantially lower than what we inferred from the $\mathrm{H} \beta$ vs. [MgFe] diagram. We note that our observations of $\mathrm{H} \gamma_{\mathrm{F}}$ and $\mathrm{H} \delta_{\mathrm{F}}$ for GCs in NGC 3115 agree well with the Lick/IDS observations of M 31 and therefore we conclude that the calibration of the models is not consistent between $\mathrm{H} \beta$ and the higher order Balmer lines. Despite this absolute calibration problem we find a good agreement in a relative sense between $\mathrm{H} \beta, \mathrm{H} \gamma_{\mathrm{F}}$ and $\mathrm{H} \delta_{\mathrm{F}}$. Therefore, at least to first order, we can say that the higher Balmer indices are not significantly affected by abundance ratios in the metallicity range probed by our data.

Comparing the distributions for MW and M 31 GCs, we find that the MW one is broader and offset towards smaller $\mathrm{H} \gamma_{\mathrm{F}}$ and $\mathrm{H} \delta_{\mathrm{F}}$ absorption consistent with the $\mathrm{H} \beta$ measurements. We ascribe this to contaminated observations for MW GCs (see above). The Lick/IDS data (particularly the $\mathrm{H} \beta$ vs. [MgFe] diagram), suggest that perhaps $\sim 3$ metal rich M 31 GCs have younger ages (3-5 Gyr). Alternatively, one could account for the strong $\mathrm{H} \beta$ absorption in these metal rich clusters if the $\mathrm{H} \beta$ index is significantly influenced by an extended blue horizontal branch in an otherwise old, metal rich stellar population. Maraston \& Thomas (2000) show that this effect can play a role in metal poor stellar populations, however, to date there is scarce observational evidence for the existence of a populous extended blue horizontal branch in metal rich clusters. Rich et al. (1997) detected a blue horizontal branch in two metal rich MW GCs and Ferraro et al. (2001) detected UV-excess stars in the core of 47 Tuc (see also Moehler et al. 2000).

Few spectroscopic observations of GCs in early-type galaxies with sufficient $S / N$ to investigate these effects have been published. Forbes et al. (2001) find that most of their $10 \mathrm{GCs}$ in the giant elliptical NGC 1399 are old and compatible with a model age of $11 \mathrm{Gyr}$ (using models by Maraston 2002, in preparation). Only two GCs display such large $\mathrm{H} \beta$ values that these have either a very young age of $\sim 2$ Gyr or are "contaminated" by a significant blue horizontal branch population which causes large $\mathrm{H} \beta$ absorption. The authors prefer the first interpretation. Larsen et al. (2002) present spectra of 14 GCs in the Sombrero Galaxy (NGC 4594). Their analysis of the co-added spectra of metal-poor and metal-rich GCs leads to age estimates between $10-15$ Gyr. The majority (11 out of 14 GCs) of the spectroscopic sample of Schroder et al. (2002) of M 81 GCs is compatible with old ages (using models by Worthey 1994). There is only one outlier with a very high $\mathrm{H} \beta$ line strength.

In summary we conclude from our best calibrated diagram of $\mathrm{H} \beta$ vs. $[\mathrm{MgFe}]$ that the majority of our sample of GCs in NGC 3115, regardless of their metallicity, are consistent with an age of $\approx 12$ Gyr. Only one, metal rich cluster (Slitlet ID: 7) shows a combination of $\mathrm{H} \beta$ and $[\mathrm{MgFe}]$ absorption strength which indicates an age lower than 8 Gyr. The higher order Balmer lines indicate a narrow distribution in age, with a hint of the metal rich clusters being younger by $\approx 2$ Gyr. The unknown dependence of the higher order Balmer lines on abundance ratios makes this age difference highly speculative. The absolute ages indicated by the higher order Balmer lines are lower compared to the $\mathrm{H} \beta$ index. We ascribe this age difference to an inaccurate calibration of the higher order Balmer lines in the current stellar population models. The Lick/IDS samples of MW and M 31 GCs also show old stellar populations; only 3 GCs in M 31 show tentative evidence of younger stars.

\subsection{Photometric versus spectroscopic metallicity estimates}

In this section we compare our spectroscopic metallicity estimates with photometric methods and also investigate the general distribution of metallicities. For this purpose we assume an average age of the GCs in NGC 3115 of 12 Gyr which is consistent with our findings in the previous section.

Figure 10a shows the purely empirical relation between $(V-I)$ colour and our mean metallicity indicator [MgFe]. There is a tight relation over the observed parameter space. Overplotted as solid line are model predictions by Maraston (2002, in preparation) and Thomas et al. (2002a) for a constant age of $12 \mathrm{Gyr}$, which is in excellent agreement with our data. We note that the model predictions for colours do not include the effects of non-solar abundance ratios.

In order to convert the colours into metallicity estimates several authors have derived linear conversion formulae based on observations for MW GCs. For example, Kundu \& Whitmore (1998) conclude that $[\mathrm{Fe} / \mathrm{H}]=-5.89+4.72(V-I)$ is a good linear approximation. One can also use the predictions of stellar population models (Maraston 2002, in preparation) to predict the relation between $(V-I)$ colour and metallicity $[\mathrm{Fe} / \mathrm{H}]$. A comparison of the empirical and synthetic calibration (12 Gyr model) is shown in Fig. $10 \mathrm{~b}$. 
Overall, the agreement is acceptable, although there are significant differences. Specifically at the low metalicity end the models predict a shallower trend than the empirical relation. In order to stay consistent with the Kundu \& Whitmore (1998) paper we use their relation to convert $(V-I)$ colour to metallicity (see also Table 1). Furthermore we determine metallicity estimates from our spectra by using the $[\mathrm{MgFe}]$ index in conjunction with the model predictions by Thomas et al. (2002a) and assuming a constant age of 12 Gyr. The comparison between photometric and spectroscopic metallicity estimates is shown in Fig. 10c.

We find a good linear relation between both methods. The best fitting linear relation including the observational errors is $[\mathrm{Fe} / \mathrm{H}]_{\text {phot }}=-0.26( \pm 0.05)+0.95( \pm 0.08) \times[\mathrm{Fe} / \mathrm{H}]_{\text {spec }}$ with a $\chi^{2}$ probability of 0.30 . The systematic offset of approximately $[\mathrm{Fe} / \mathrm{H}]=-0.26$ in the sense that the spectroscopic metallicity measurements are larger is consistent with the difference between model predictions and empirical calibration of the conversion formulae between colour and $[\mathrm{Fe} / \mathrm{H}]$ as shown in Fig. 10b. The predicted non-linearity of $(V-I)$ colour as function of metallicity below $[\mathrm{Fe} / \mathrm{H}]=-1.5$ cannot be tested since our data do not really cover this range.

In summary we confirm with our accurate spectroscopic observations that the bimodal colour distribution seen in NGC 3115 GCs is dominated by a metallicity effect rather than by an age difference. Furthermore, both $(V-I)$ colour peaks do show a substantial spread in metallicity. We conclude that in the metallicity range $-1.5 \leq[\mathrm{Fe} / \mathrm{H}] \leq 0.0$ and in absence of young GCs, the $(V-I)$ colour is indeed a good indicator for metallicity. We note that for metallicities below $[\mathrm{Fe} / \mathrm{H}]=-1.5$ this may not be the case anymore.

\section{Discussion}

Before we discuss the implications of our results it needs to be noted that our sample of GCs in NGC 3115 is dominated by the bright end of the luminosity function. Our faintest object (Slitlet ID: 17) which we could use for the line-strength analysis is 0.9 mag brighter than the peak of the GC luminosity function. Furthermore, since this project was only a pilot study we observed only a small number of clusters over a limited region across NGC 3115. Although our data can give clear insights into the globular cluster system of NGC 3115 it is not necessarily a representative sample. The comparison with the Lick/IDS observations of MW and M31 GCs needs to be viewed with caution since these samples cover a larger range in absolute GC magnitude.

Using their HST $V, I$ photometry Kundu \& Whitmore (1998) develop the following formation scenario for NGC 3115. The blue, metal poor clusters are formed with the halo/bulge component of the galaxy very early on. Then about $4 \pm 3$ Gyr later an unequal mass, gas-rich merger event forms the disk component and the associated red, metal rich clusters. The authors point out that the spatial distribution of the blue and red clusters are consistent with the halo/bulge and disk components respectively. Furthermore, there is evidence from optical imaging (Silva et al. 1989), that the disk is bluer and therefore perhaps younger than the halo/bulge component, consistent with the above outlined scenario.

In our spectroscopic study of the GCs in NGC 3115 we find that the clusters are consistent with being coeval at about 11$12 \mathrm{Gyr}$. There is perhaps a weak hint of the red clusters being younger, but by no more than $\sim 2$ Gyr. This on its own would not rule out the scenario by Kundu \& Whitmore (1998), however our estimates of the abundance ratios show that at least the population of red, metal rich clusters is not homogeneous. For these objects we find a range in abundance ratios from solar to about $[\mathrm{Mg} / \mathrm{Fe}] \simeq 0.5$. In our small sample of limited spatial extend we do not find any clear trends of the chemical composition of GCs with the kinematics (see Fig. 3). The relative velocities of most of the clusters in our sample are consistent with rotation. A larger sample of more complete spatial coverage is needed to establish possible trends between abundance ratios and e.g., age, metallicity, spatial position and kinematics.

What we can however say is that the metal-rich clusters with solar abundance ratios must have been made out of well mixed material which incorporates the products of both SN II (the main producer of alpha elements) and SN Ia (the main producer of Fe-peak elements). Since SN Ia are somewhat delayed compared to SN II the solar abundance ratio clusters must have formed after the initial star burst in NGC 3115. There are many possible scenarios to explain the observed abundance ratio distribution, but it is hard to fit them into a simple picture of only two distinct formation events which create the red and blue globular cluster sub-populations (see Beasley et al. 2002a).

One scenario which we would like to put forward for further discussion is the following. The metal poor (blue), non-solar abundance ratio clusters are associated with the halo formation, and the metal-rich (red), non-solar abundance ratio clusters are formed together with the bulge as was similarly proposed for the Milky Way by Carney et al. (1990) and Wyse \& Gilmore (1992). The metal rich, solar ratio clusters are then formed with the disk of this lenticular galaxy $\sim 1-2$ Gyr after the initial star-burst perhaps in connection with a merger. This scenario would then require the disk to have also close to solar abundance ratios, which can be tested by observations of the integrated light. Furthermore, if this scenario is correct the spatial distribution and kinematics of disk GCs will be distinct from the population of halo and bulge GCs in NGC 3115. Future, larger samples of NGC 3115 GCs will be very valuable to explore the connection between disk formation and metal rich globular cluster formation.

More spectroscopic observations of GCs in nearby galaxies of various types will be very valuable to improve our stellar population models and learn more about the early starformation epochs in early-type galaxies. However, the mismatch of the models and some observed indices demonstrates that we are also in urgent need for a new, high-quality fluxcalibrated spectral library in order to exclude simple observational offsets. Only then can we make good progress with improvements on the input physics of stellar population models and their application to extragalactic objects. 

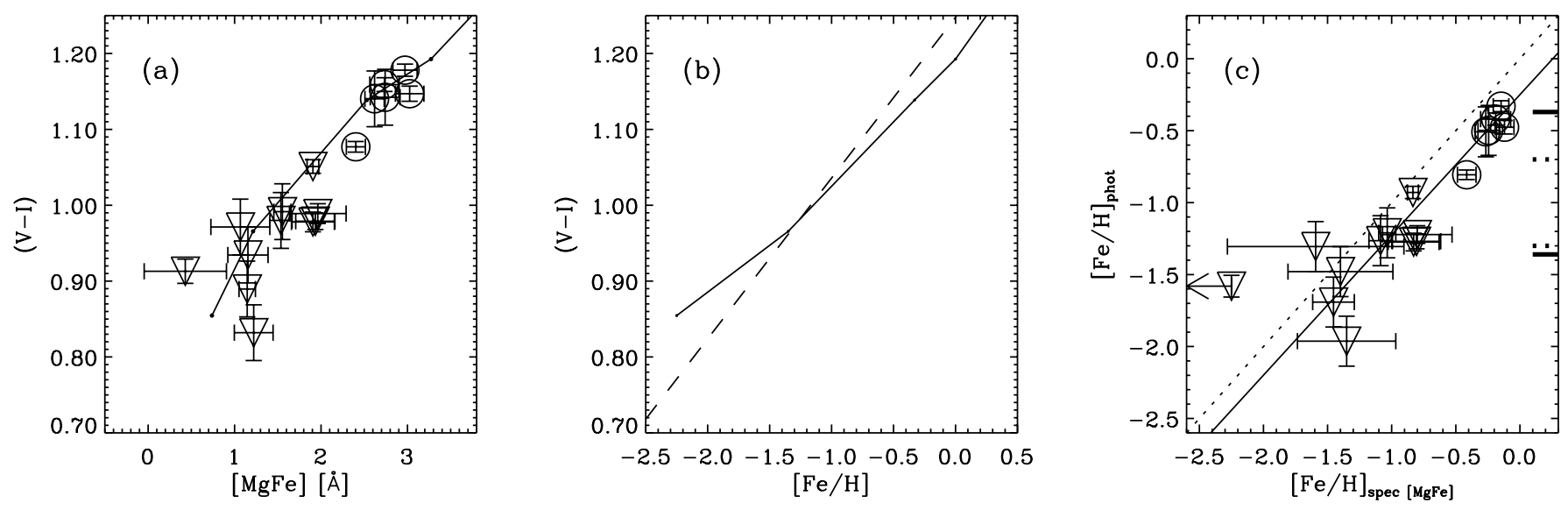

Fig. 10. Comparison of photometric and spectroscopic metallicity estimates. In the left panel the $[\mathrm{MgFe}]$ index is plotted against (V-I) colours (including the pseudo $(V-I)$ colours derived from the spectra). Overplotted as solid line is the prediction for a 12 Gyr stellar population model by Maraston (2002, in preparation) and Thomas et al. (2002a). In the middle panel we show a comparison between an empirical calibration of $(V-I)$ colour against metallicity (dashed line; Kundu \& Whitmore 1998) and predictions from stellar population models (solid line, 12 Gyr; Maraston 2002, in preparation). In the right panel, photometric metallicities are calculated according to Kundu \& Whitmore (1998), while spectroscopic metallicities are derived from the $[\mathrm{MgFe}]$ index in comparison to models of Thomas et al. (2002a) assuming a constant age of 12 Gyr. The data point at $[\mathrm{Fe} / \mathrm{H}]_{\text {spec }}[\mathrm{MgFe}]=-2.25$ represents a globular cluster with line-strength weaker than the currently available model predictions and is therefore only an upper limit. The lines at the right hand side of the plot show the peak positions of the bimodal distributions of GCs observed by Kundu \& Whitmore (1998, solid lines) and RGB stars observed by Elson (1997, dotted lines).

\section{Conclusions}

We present new, accurate measurements of absorption linestrength indices of 17 globular clusters (hereafter GCs) in the nearby S0 galaxy NGC 3115. Our objects span a range in colour so that the bimodal $(V-I)$ colour distribution is well sampled.

A critical comparison with Lick/IDS data (Trager et al. 1998) of GCs in M 31 and the Milky Way (hereafter MW) is presented. The Lick/IDS measurements of the $\mathrm{H} \gamma_{\mathrm{A}, \mathrm{F}}$ and $\mathrm{H} \delta_{\mathrm{A}, \mathrm{F}}$ indices for MW and M31 GCs are presented for the first time in this paper. The data are analysed with new stellar population models (Thomas et al. 2002a) which are able to predict linestrength not only as a function of age and metallicity but also as a function of abundance ratio. Specifically, abundance ratio biases in the stellar library, which is an essential ingredient to the model predictions, have been taken into account for the first time.

Our main results are listed in the following:

- The GCs in NGC 3115 show a range of abundance ratios as estimated by the strength of $\mathrm{Mg}$ and Fe lines. Specifically we find for both red and blue clusters solar as well as supersolar values (up to $[\mathrm{Mg} / \mathrm{Fe}] \simeq+0.5$ ). Lick/IDS data of M 31 GCs show a similar distribution while MW GCs are consistent with a constant value of $[\mathrm{Mg} / \mathrm{Fe}] \simeq 0.3$. The latter is in agreement with recent studies of the resolved stellar populations in MW GCs.

- Our analysis of $\mathrm{H} \beta, \quad \mathrm{H} \gamma_{\mathrm{F}}$ and $\mathrm{H} \delta_{\mathrm{F}} \quad$ vs. $[\mathrm{MgFe}]$ age/metallicity diagnostic diagrams shows that the red and blue GC populations are coeval within $\approx 2 \mathrm{Gyr}$. Our best calibrated diagram $(\mathrm{H} \beta,[\mathrm{MgFe}])$ indicates a mean age of 11-12 Gyr. However, the higher order Balmer lines, although confirming the similar ages of blue and red clusters, indicate younger absolute ages ( $\approx 6 \mathrm{Gyr})$. We ascribe these younger age estimates to inaccurate model calibrations of the higher order Balmer lines. Evidence for the existence of young ( $<5 \mathrm{Gyr}) \mathrm{GCs}$ in the studied galaxies is scarce. Only one cluster in NGC 3115 and perhaps 3 clusters in M 31 show a combination of Balmer and metal absorption strength which is consistent with such young ages. The strong $\mathrm{H} \beta$ absorption can be alternatively explained if these clusters have old stellar populations with a populous extended horizontal branch.

- We present a comparison of photometric and spectroscopic metallicity determinations and find a good linear relation in the metallicity range probed by our sample of NGC 3115 clusters $(-1.5<[\mathrm{Fe} / \mathrm{H}]<0.0)$. The photometric estimates are systematically lower $(\simeq-0.26)$ in comparison with our spectroscopic measurements. We note that our observations clearly show that each colour peak has a significant spread in metallicity rather than being consistent with a narrow distribution.

- The existence of solar as well as elevated Mg-to-Fe ratios at a given metallicity for GCs in NGC 3115 indicates that a simple scenario of two distinct star-formation episodes is not sufficient to explain the formation of this galaxy. Probably a realistic model needs to incorporate more than two distinct star-formation events.

- We detect a clear signal of rotation in our sample of GCs independent of their metallicities.

Larger samples of high signal-to-noise spectra of GCs in nearby galaxies are required to increase our knowledge of how these galaxies formed. Particularly the measurement of abundance ratios in extragalactic GCs is now possible and will 
Table A.1. Line-strength measurements for NGC 3115 globular clusters.

\begin{tabular}{|c|c|c|c|c|c|c|c|c|c|c|}
\hline Slitlet ID & $\begin{array}{r}\mathrm{H} \delta_{\mathrm{A}} \\
{[\AA]}\end{array}$ & $\begin{array}{r}\mathrm{H} \delta_{\mathrm{F}} \\
{[\AA]}\end{array}$ & $\begin{array}{r}\mathrm{CN}_{1} \\
{[\mathrm{mag}]}\end{array}$ & $\begin{array}{r}\mathrm{CN}_{2} \\
{[\mathrm{mag}]}\end{array}$ & $\begin{array}{r}\mathrm{Ca} 4227 \\
{[\AA]}\end{array}$ & $\begin{array}{r}\mathrm{G} 4300 \\
{[\AA ̊]}\end{array}$ & $\begin{array}{r}\mathrm{H} \gamma_{\mathrm{A}} \\
{[\AA]}\end{array}$ & $\begin{array}{r}\mathrm{H} \gamma_{\mathrm{F}} \\
{[\AA]}\end{array}$ & $\begin{array}{r}\mathrm{Fe} 4383 \\
{[\AA]}\end{array}$ & $\begin{array}{r}\mathrm{Ca} 4455 \\
{[\AA]}\end{array}$ \\
\hline \multirow[t]{2}{*}{3} & -1.41 & 0.32 & 0.049 & 0.076 & 0.95 & 4.64 & -4.76 & -0.92 & 3.74 & 1.05 \\
\hline & 0.54 & 0.37 & 0.015 & 0.018 & 0.26 & 0.43 & 0.51 & 0.31 & 0.59 & 0.29 \\
\hline \multirow[t]{2}{*}{5} & 1.44 & 1.81 & -0.014 & 0.022 & 0.54 & 2.84 & -0.92 & 1.04 & 2.03 & 0.40 \\
\hline & 0.34 & 0.23 & 0.010 & 0.012 & 0.18 & 0.30 & 0.32 & 0.18 & 0.44 & 0.21 \\
\hline \multirow[t]{2}{*}{7} & -1.00 & 0.29 & 0.078 & 0.120 & 1.41 & 4.45 & -4.60 & -0.53 & 3.81 & 0.67 \\
\hline & 0.46 & 0.32 & 0.013 & 0.015 & 0.21 & 0.35 & 0.38 & 0.23 & 0.49 & 0.22 \\
\hline \multirow[t]{2}{*}{8} & 0.49 & 1.38 & 0.006 & 0.038 & 0.83 & 3.29 & -1.92 & 0.54 & 2.36 & 0.56 \\
\hline & 0.18 & 0.12 & 0.005 & 0.006 & 0.09 & 0.15 & 0.16 & 0.10 & 0.22 & 0.11 \\
\hline \multirow[t]{2}{*}{9} & 0.68 & 1.27 & 0.015 & 0.054 & 0.79 & 3.91 & -3.15 & -0.17 & 3.09 & 0.73 \\
\hline & 0.28 & 0.20 & 0.008 & 0.009 & 0.14 & 0.23 & 0.27 & 0.17 & 0.35 & 0.17 \\
\hline \multirow[t]{2}{*}{12} & 2.42 & 2.87 & 0.010 & 0.067 & 0.60 & 2.38 & -0.39 & 1.24 & 2.53 & 0.77 \\
\hline & 0.52 & 0.34 & 0.016 & 0.019 & 0.27 & 0.49 & 0.51 & 0.30 & 0.71 & 0.35 \\
\hline \multirow[t]{2}{*}{13} & 2.02 & 1.95 & -0.011 & 0.043 & 0.94 & 2.64 & -1.78 & 1.20 & 2.62 & 0.04 \\
\hline & 0.81 & 0.57 & 0.023 & 0.028 & 0.42 & 0.72 & 0.81 & 0.49 & 1.08 & 0.53 \\
\hline \multirow[t]{2}{*}{$14 \mathrm{~b}$} & 0.75 & 1.23 & 0.012 & 0.035 & 1.01 & 3.93 & -4.56 & -0.58 & 3.89 & 1.26 \\
\hline & 0.50 & 0.36 & 0.015 & 0.018 & 0.25 & 0.40 & 0.46 & 0.28 & 0.60 & 0.28 \\
\hline \multirow[t]{2}{*}{15} & 2.54 & 1.96 & -0.015 & 0.020 & 1.16 & 3.29 & -1.72 & 0.81 & 3.00 & 0.12 \\
\hline & 0.64 & 0.45 & 0.019 & 0.023 & 0.34 & 0.56 & 0.64 & 0.38 & 0.82 & 0.42 \\
\hline \multirow[t]{2}{*}{16} & 3.50 & 2.95 & -0.063 & -0.058 & -0.21 & 0.55 & 0.96 & 2.24 & 2.07 & 0.60 \\
\hline & 0.84 & 0.55 & 0.025 & 0.030 & 0.45 & 0.83 & 0.78 & 0.50 & 1.11 & 0.54 \\
\hline \multirow[t]{2}{*}{17} & -0.27 & 0.14 & 0.056 & 0.080 & 1.07 & 5.02 & -4.59 & -1.11 & 3.53 & 0.47 \\
\hline & 0.54 & 0.37 & 0.014 & 0.018 & 0.24 & 0.40 & 0.48 & 0.29 & 0.57 & 0.28 \\
\hline \multirow[t]{2}{*}{21} & 2.01 & 2.63 & -0.048 & -0.034 & 0.70 & 3.34 & -0.17 & 2.03 & 1.85 & 0.17 \\
\hline & 0.85 & 0.59 & 0.024 & 0.029 & 0.43 & 0.79 & 0.79 & 0.48 & 1.11 & 0.54 \\
\hline \multirow[t]{2}{*}{23} & -1.79 & 0.40 & 0.069 & 0.099 & 0.90 & 4.38 & -3.97 & -0.21 & 4.77 & 1.13 \\
\hline & 0.37 & 0.24 & 0.010 & 0.012 & 0.16 & 0.28 & 0.31 & 0.19 & 0.37 & 0.20 \\
\hline \multirow[t]{2}{*}{24} & 1.99 & 2.06 & -0.008 & 0.012 & 0.83 & 2.87 & -1.23 & 0.93 & 2.45 & 0.32 \\
\hline & 0.24 & 0.17 & 0.008 & 0.009 & 0.12 & 0.22 & 0.24 & 0.14 & 0.32 & 0.15 \\
\hline \multirow[t]{2}{*}{$25 a$} & 1.41 & 1.53 & -0.049 & -0.041 & 0.32 & 2.66 & -0.31 & 0.77 & 0.46 & 0.38 \\
\hline & 0.54 & 0.36 & 0.015 & 0.019 & 0.28 & 0.49 & 0.51 & 0.32 & 0.71 & 0.33 \\
\hline \multirow[t]{2}{*}{$25 b$} & 2.33 & 2.48 & -0.059 & -0.034 & 0.49 & 2.33 & 0.30 & 1.79 & 1.27 & 0.31 \\
\hline & 0.22 & 0.15 & 0.006 & 0.008 & 0.11 & 0.19 & 0.21 & 0.13 & 0.31 & 0.15 \\
\hline \multirow[t]{2}{*}{$26 b$} & 2.46 & 2.07 & -0.064 & -0.047 & 0.54 & 1.99 & 1.35 & 2.19 & 1.33 & 0.03 \\
\hline & 0.53 & 0.35 & 0.016 & 0.019 & 0.28 & 0.49 & 0.49 & 0.30 & 0.71 & 0.34 \\
\hline
\end{tabular}

Notes: Listed are line-strength measurements in the wavelength range 4000-4500 A for globular clusters in NGC 3115 (see also Table 1). The first line for each object shows the measured index values whereas the second line lists the associated errors. The unit for each index is given at the top in square brackets.

deliver important new constraints for galaxy formation models which are not accessible with broad-band colours alone.

Acknowledgements. Part of this work was supported by the Volkswagen Foundation (I/76520). We thank Dr. J. Heidt (Heidelberg) and the ESO Paranal staff for the efficient observations, the resulting data of which were the basis for this Paper. We are also very grateful to D. Thomas, C. Maraston and R. Bender who provided their models prior to publication. We thank the referee B. W. Carney for a quick and helpful referee report.

\section{Appendix A: Tables of line-strength measurements}

\section{A.1. Line-strength measurements for globular clusters in NGC 3115}

Tables A.1 and A.2 present the line-strength measurements for globular clusters in NGC 3115. The spectra have been broadened to the Lick/IDS resolution prior to index measurements. Small offsets have been made to match the Lick system as 
Table A.2. Lick/IDS line-strength measurements for NGC 3115 globular clusters.

\begin{tabular}{|c|c|c|c|c|c|c|c|c|c|c|}
\hline Slitlet ID & $\begin{array}{r}\mathrm{Fe} 4531 \\
{[\AA]}\end{array}$ & $\begin{array}{r}\mathrm{C}_{2} 4668 \\
[\AA]]\end{array}$ & $\begin{array}{l}\mathrm{H} \beta \\
[\AA]]\end{array}$ & $\begin{array}{r}\mathrm{Fe} 5015 \\
{[\AA]}\end{array}$ & $\begin{array}{r}\mathrm{Mg}_{1} \\
{[\mathrm{mag}]}\end{array}$ & $\begin{array}{r}\mathrm{Mg}_{2} \\
{[\mathrm{mag}]}\end{array}$ & $\begin{array}{r}\operatorname{Mg} b \\
{[\AA]}\end{array}$ & $\begin{array}{r}\mathrm{Fe} 5270 \\
{[\AA]}\end{array}$ & $\begin{array}{r}\text { Fe5335 } \\
{[\AA]}\end{array}$ & $\begin{array}{r}\mathrm{Fe} 5406 \\
{[\AA]}\end{array}$ \\
\hline \multirow[t]{2}{*}{3} & 2.94 & 3.88 & 1.59 & 5.24 & 0.068 & 0.216 & 3.59 & 2.30 & 1.90 & 1.46 \\
\hline & 0.44 & 0.65 & 0.25 & 0.56 & 0.006 & 0.007 & 0.28 & 0.28 & 0.31 & 0.23 \\
\hline \multirow[t]{2}{*}{5} & 1.87 & 1.45 & 2.12 & 3.51 & 0.038 & 0.105 & 1.86 & 1.49 & 1.05 & 0.68 \\
\hline & 0.34 & 0.49 & 0.19 & 0.41 & 0.005 & 0.005 & 0.20 & 0.22 & 0.25 & 0.19 \\
\hline \multirow[t]{2}{*}{7} & 3.20 & 4.07 & 2.12 & 5.90 & 0.064 & 0.196 & 3.84 & 2.03 & 2.57 & 1.74 \\
\hline & 0.35 & 0.53 & 0.20 & 0.43 & 0.005 & 0.006 & 0.22 & 0.24 & 0.25 & 0.20 \\
\hline \multirow[t]{2}{*}{8} & 2.20 & 1.87 & 2.18 & 3.20 & 0.025 & 0.110 & 2.04 & 1.83 & 1.73 & 0.89 \\
\hline & 0.17 & 0.25 & 0.10 & 0.23 & 0.002 & 0.003 & 0.10 & 0.12 & 0.13 & 0.11 \\
\hline \multirow[t]{2}{*}{9} & 2.58 & 2.89 & 1.71 & 4.13 & 0.050 & 0.160 & 3.32 & 1.97 & 1.51 & 0.83 \\
\hline & 0.26 & 0.39 & 0.16 & 0.34 & 0.004 & 0.004 & 0.16 & 0.18 & 0.20 & 0.16 \\
\hline \multirow[t]{2}{*}{12} & 0.70 & -0.29 & 2.33 & 3.53 & 0.025 & 0.118 & 2.42 & 1.88 & 1.21 & 1.21 \\
\hline & 0.61 & 0.86 & 0.32 & 0.69 & 0.008 & 0.010 & 0.35 & 0.37 & 0.43 & 0.34 \\
\hline \multirow[t]{2}{*}{13} & 1.99 & 1.99 & 1.38 & 2.59 & 0.014 & 0.107 & 2.12 & 2.73 & 0.90 & 0.46 \\
\hline & 0.87 & 1.30 & 0.49 & 1.05 & 0.011 & 0.013 & 0.52 & 0.54 & 0.63 & 0.52 \\
\hline \multirow[t]{2}{*}{$14 \mathrm{~b}$} & 3.26 & 1.87 & 1.38 & 3.75 & 0.052 & 0.174 & 2.90 & 2.45 & 2.69 & 1.22 \\
\hline & 0.43 & 0.67 & 0.26 & 0.57 & 0.006 & 0.007 & 0.27 & 0.28 & 0.31 & 0.26 \\
\hline \multirow[t]{2}{*}{15} & 1.68 & -0.36 & 1.31 & 3.85 & 0.035 & 0.125 & 2.25 & 1.36 & 1.87 & 0.47 \\
\hline & 0.66 & 1.00 & 0.37 & 0.80 & 0.009 & 0.010 & 0.39 & 0.41 & 0.48 & 0.41 \\
\hline \multirow[t]{2}{*}{16} & 1.64 & -1.13 & 2.65 & 1.62 & 0.015 & 0.028 & 0.56 & 0.36 & $0.30^{a}$ & -0.28 \\
\hline & 0.92 & 1.48 & 0.56 & 1.18 & 0.013 & 0.015 & 0.57 & 0.65 & 1.12 & 0.57 \\
\hline \multirow[t]{2}{*}{17} & 3.18 & 3.47 & 1.72 & 4.80 & 0.055 & 0.199 & 3.79 & 2.24 & 2.59 & 1.19 \\
\hline & 0.43 & 0.66 & 0.26 & 0.52 & 0.006 & 0.007 & 0.26 & 0.28 & 0.30 & 0.25 \\
\hline \multirow[t]{2}{*}{21} & 0.66 & 0.26 & 1.39 & 1.48 & 0.009 & 0.070 & 1.07 & 0.99 & 1.13 & 1.11 \\
\hline & 0.92 & 1.37 & 0.53 & 1.11 & 0.012 & 0.013 & 0.53 & 0.58 & 0.63 & 0.53 \\
\hline \multirow[t]{2}{*}{23} & 3.21 & 2.36 & 1.78 & 4.31 & 0.036 & 0.166 & 2.82 & 2.68 & 2.19 & 1.65 \\
\hline & 0.30 & 0.45 & 0.18 & 0.36 & 0.004 & 0.005 & 0.18 & 0.18 & 0.21 & 0.16 \\
\hline \multirow[t]{2}{*}{24} & 2.01 & 0.46 & 1.96 & 3.04 & 0.023 & 0.103 & 1.71 & 1.51 & 1.31 & 1.06 \\
\hline & 0.25 & 0.39 & 0.15 & 0.32 & 0.003 & 0.004 & 0.16 & 0.17 & 0.19 & 0.15 \\
\hline \multirow[t]{2}{*}{$25 \mathrm{a}$} & 0.12 & -0.86 & 2.57 & 0.97 & -0.004 & 0.037 & 1.44 & 1.29 & 0.78 & -0.33 \\
\hline & 0.58 & 0.87 & 0.32 & 0.81 & 0.008 & 0.009 & 0.33 & 0.38 & 0.46 & 0.36 \\
\hline \multirow[t]{2}{*}{$25 b$} & 1.60 & 0.61 & 2.23 & 2.66 & 0.016 & 0.063 & 1.15 & 1.06 & 1.23 & 0.50 \\
\hline & 0.23 & 0.33 & 0.13 & 0.30 & 0.003 & 0.004 & 0.14 & 0.17 & 0.18 & 0.14 \\
\hline \multirow[t]{2}{*}{$26 \mathrm{~b}$} & 3.07 & 0.05 & 2.15 & 3.41 & 0.027 & 0.088 & 0.99 & 1.52 & 1.18 & 0.76 \\
\hline & 0.54 & 0.80 & 0.32 & 0.66 & 0.007 & 0.009 & 0.35 & 0.37 & 0.39 & 0.32 \\
\hline
\end{tabular}

Notes: Listed are line-strength measurements in the wavelength range 4500-5450 ̊̊ for globular clusters in NGC 3115 (see also Table 1). The first line for each object shows the measured index values whereas the second line lists the associated errors. The unit for each index is given at the top in square brackets.

${ }^{a}$ For this globular cluster we measured a Fe5335 index of $-1.24( \pm 0.75) \AA$. This negative number is caused by a noisy section in the spectrum. By comparing this index with other Fe indices we established that a realistic value of the Fe 5335 index for this globular cluster should be +0.3 . To reflect the unertainty in this procedure we increased the error by a factor of 1.5 .

closely as possible (see Sect. 2 for details). For a summary of the definition of Lick/IDS indices see Trager et al. (1998).

\section{A.2. Lick/IDS observations of $H \gamma_{A, F}$ and $H \delta_{A, F}$ indices for Milky Way and M31 globular clusters}

Since the higher order Balmer indices of Milky Way and M 31 globular clusters as measured in the Lick/IDS system have not yet been published, we list the measurements of $\mathrm{H} \gamma_{\mathrm{A}, \mathrm{F}}$ and $\mathrm{H} \delta_{\mathrm{A}, \mathrm{F}}$ in Tables A.3 and A.4. The Lick observations are described in Burstein et al. (1984), names are explained there (see also Trager et al. 1998). M 31 globular clusters were observed with the standard aperture of the Lick/IDS system $\left(1{ }^{\prime \prime} 4 \times 4\right.$ ".'0). The MW globular clusters were observed with a long-slit of standard width that was raster-scanned on the sky to create a square aperture of size $66^{\prime \prime} \times 66^{\prime \prime}$ (only for NGC 6624 the aperture is $\left.45^{\prime \prime} \times 60^{\prime \prime}\right)$. Raster scans have the same spectral resolution as standard slit width scans. For the definition of the higher order Balmer indices see Worthey \& Ottaviani (1997). 
Table A.3. Lick/IDS line-strength measurements for Milky Way globular clusters.

\begin{tabular}{|c|c|c|c|c|}
\hline Name & $\begin{array}{r}\mathrm{H} \delta_{\mathrm{A}} \\
{[\AA]}\end{array}$ & $\begin{array}{r}\mathrm{H} \delta_{\mathrm{F}} \\
[\AA]]\end{array}$ & $\begin{array}{r}\mathrm{H} \gamma_{\mathrm{A}} \\
{[\AA]}\end{array}$ & $\begin{array}{r}\mathrm{H} \gamma_{\mathrm{F}} \\
{[\AA]}\end{array}$ \\
\hline \multirow[t]{2}{*}{ NGC 5024} & 4.56 & 3.01 & \multirow[t]{2}{*}{$\ldots$} & 2.34 \\
\hline & 1.20 & 0.75 & & 0.62 \\
\hline \multirow[t]{2}{*}{ NGC 5272} & 2.88 & 2.30 & \multirow[t]{2}{*}{$\ldots$} & 1.77 \\
\hline & 0.88 & 0.55 & & 0.45 \\
\hline \multirow[t]{2}{*}{ NGC 5904} & 3.57 & 2.67 & 1.34 & 2.09 \\
\hline & 1.22 & 0.76 & 0.92 & 0.63 \\
\hline \multirow[t]{2}{*}{ NGC 6171} & 2.18 & 1.73 & \multirow[t]{2}{*}{$\ldots$} & -0.60 \\
\hline & 1.95 & 1.22 & & 1.00 \\
\hline \multirow[t]{2}{*}{ NGC 6205} & 2.68 & 2.38 & 1.04 & 1.79 \\
\hline & 0.54 & 0.34 & 0.41 & 0.28 \\
\hline \multirow[t]{2}{*}{ NGC 6218} & 3.83 & 3.17 & \multirow[t]{2}{*}{$\ldots$} & 1.96 \\
\hline & 1.41 & 0.88 & & 0.73 \\
\hline \multirow[t]{2}{*}{ NGC 6229} & 3.57 & 2.35 & 0.79 & 1.14 \\
\hline & 1.72 & 1.07 & 1.29 & 0.88 \\
\hline \multirow{2}{*}{ NGC 6341} & 3.65 & 2.87 & 1.72 & 2.33 \\
\hline & 0.69 & 0.43 & 0.52 & 0.36 \\
\hline \multirow[t]{2}{*}{ NGC 6356} & $\ldots$ & 0.01 & \multirow[t]{2}{*}{$\ldots$} & -1.16 \\
\hline & & 0.66 & & 0.55 \\
\hline \multirow[t]{2}{*}{ NGC 6624} & -1.26 & 0.73 & -4.70 & -0.24 \\
\hline & 1.02 & 0.64 & 0.76 & 0.52 \\
\hline \multirow[t]{2}{*}{ NGC 6637} & -1.37 & -0.17 & -4.23 & -1.70 \\
\hline & 0.90 & 0.56 & 0.68 & 0.47 \\
\hline \multirow[t]{2}{*}{ NGC 6712} & 3.18 & 2.42 & 0.64 & 0.25 \\
\hline & 1.87 & 1.17 & 1.40 & 0.96 \\
\hline \multirow[t]{2}{*}{ NGC 6838} & 0.66 & 0.69 & -1.39 & 0.35 \\
\hline & 1.32 & 0.83 & 0.99 & 0.68 \\
\hline \multirow[t]{2}{*}{ NGC 6981} & 3.24 & 2.55 & \multirow[t]{2}{*}{$\ldots$} & 1.45 \\
\hline & 1.84 & 1.15 & & 0.95 \\
\hline \multirow[t]{2}{*}{ NGC 7006} & 3.45 & 4.37 & \multirow[t]{2}{*}{$\cdots$} & 1.64 \\
\hline & 1.57 & 0.98 & & 0.81 \\
\hline \multirow[t]{2}{*}{ NGC 7078} & 3.37 & 2.69 & 1.85 & 2.28 \\
\hline & 0.75 & 0.47 & 0.56 & 0.39 \\
\hline \multirow[t]{2}{*}{ NGC 7089} & 2.71 & 2.03 & 1.19 & 2.24 \\
\hline & 0.92 & 0.57 & 0.69 & 0.47 \\
\hline
\end{tabular}

Notes: We list here the Lick/IDS measurements of the indices $\mathrm{H} \delta_{\mathrm{A}}$, $\mathrm{H} \delta_{\mathrm{F}}, \mathrm{H} \gamma_{\mathrm{A}}$ and $\mathrm{H} \gamma_{\mathrm{F}}$ for MW globular clusters. The Lick/IDS system is described in detail in Trager et al. (1998). For the definition of the higher order Balmer indices see Worthey \& Ottaviani (1997). The first line for each object shows the measured index values whereas the second line lists the associated errors. The unit for each index is given at the top in square brackets. Three dots indicate where no index measurement is available.

\section{References}

Ashman, K. M., \& Zepf, S. E. 1992, ApJ, 384, 50

Ashman, K. M., \& Zepf, S. E. 1998, Globular cluster systems (Cambridge University Press)

Beasley, M. A., Baugh, C. M., Forbes, D. A., Sharples, R. M., \& Frenk, C. S. 2002a, MNRAS, 333, 383

Beasley, M. A., Hoyle, F., \& Sharples, R. M. 2002b, MNRAS, 336,168

Bekki, K. 1998, ApJ, 502, L133

Borges, A. C., Idiart, T. P., de Freitas Pacheco, J. A., \& Thevenin, F. 1995, AJ, 110, 2408

Bridges, T. J., Ashman, K. M., Zepf, S. E., et al. 1997, MNRAS, 284, 376
Table A.4. Line-strength measurements for globular clusters in M 31 .

\begin{tabular}{|c|c|c|c|c|c|}
\hline Name1 & Name2 & $\begin{array}{r}\mathrm{H} \delta_{\mathrm{A}} \\
{[\AA]}\end{array}$ & $\begin{array}{r}\mathrm{H} \delta_{\mathrm{F}} \\
{[\AA]}\end{array}$ & $\begin{array}{r}\mathrm{H} \gamma_{\mathrm{A}} \\
{[\AA]}\end{array}$ & $\begin{array}{r}\mathrm{H} \gamma_{\mathrm{F}} \\
{[\AA]}\end{array}$ \\
\hline \multirow[t]{2}{*}{ MII } & K1 & 1.29 & 1.99 & -1.55 & 0.84 \\
\hline & & 0.54 & 0.34 & 0.40 & 0.28 \\
\hline \multirow[t]{2}{*}{ MIV } & K219 & 3.36 & 2.30 & 2.72 & 2.72 \\
\hline & & 0.60 & 0.38 & 0.45 & 0.31 \\
\hline \multirow[t]{2}{*}{ V4 } & K33 & 3.86 & 2.82 & 0.68 & 1.46 \\
\hline & & 1.47 & 0.92 & 1.10 & 0.76 \\
\hline \multirow[t]{2}{*}{ V12 } & K76 & 1.81 & 1.55 & 0.12 & 1.53 \\
\hline & & 1.00 & 0.63 & 0.75 & 0.52 \\
\hline \multirow[t]{2}{*}{ V23 } & K119 & 2.38 & 2.68 & -0.08 & 1.45 \\
\hline & & 1.06 & 0.66 & 0.79 & 0.54 \\
\hline \multirow[t]{2}{*}{ V42 } & K78 & -0.19 & 1.24 & $\ldots$ & -0.30 \\
\hline & & 0.65 & 0.41 & & 0.34 \\
\hline \multirow[t]{2}{*}{ V64 } & $\mathrm{K} 213$ & $\ldots$ & 1.88 & $\ldots$ & 0.74 \\
\hline & & & 0.65 & & 0.54 \\
\hline \multirow[t]{2}{*}{ V76 } & K263 & 2.55 & 3.13 & 0.83 & 1.92 \\
\hline & & 1.24 & 0.77 & 0.93 & 0.64 \\
\hline \multirow[t]{2}{*}{ V87 } & K222 & -2.58 & -0.83 & -3.95 & -1.12 \\
\hline & & 1.29 & 0.80 & 0.96 & 0.66 \\
\hline \multirow[t]{2}{*}{ V95 } & K229 & 3.39 & 2.71 & 0.93 & 1.97 \\
\hline & & 0.77 & 0.48 & 0.58 & 0.40 \\
\hline \multirow[t]{2}{*}{ V99 } & K286 & 3.44 & 2.33 & 0.64 & 2.01 \\
\hline & & 0.95 & 0.60 & 0.72 & 0.49 \\
\hline \multirow[t]{2}{*}{ V100 } & K217 & -2.39 & 0.34 & -5.56 & -1.12 \\
\hline & & 0.86 & 0.54 & 0.65 & 0.45 \\
\hline \multirow[t]{2}{*}{ V116 } & K244 & -3.69 & 0.03 & - & 0.20 \\
\hline & & 1.29 & 0.81 & - & 0.67 \\
\hline \multirow[t]{2}{*}{ V196 } & K302 & 2.26 & 2.43 & 0.79 & 2.07 \\
\hline & & 0.83 & 0.52 & 0.62 & 0.43 \\
\hline \multirow[t]{2}{*}{ V282 } & K280 & -1.36 & 0.09 & -4.75 & -0.22 \\
\hline & & 0.57 & 0.36 & 0.43 & 0.29 \\
\hline \multirow[t]{2}{*}{ V301 } & K64 & 3.14 & 2.74 & $\ldots$ & 1.99 \\
\hline & & 1.23 & 0.77 & & 0.63 \\
\hline \multirow[t]{2}{*}{ V101 } & K272 & $\ldots$ & 1.75 & $\ldots$ & 0.76 \\
\hline & & & 0.63 & & 0.52 \\
\hline
\end{tabular}

Notes: We list here the Lick/IDS measurements of the indices $\mathrm{H} \delta_{\mathrm{A}}$, $\mathrm{H} \delta_{\mathrm{F}}, \mathrm{H} \gamma_{\mathrm{A}}$ and $\mathrm{H} \gamma_{\mathrm{F}}$ for globular clusters in M 31 . The Lick/IDS system is described in detail in Trager et al. (1998). For the definition of the higher order Balmer indices see Worthey \& Ottaviani (1997). The first line for each object shows the measured index values whereas the second line lists the associated errors. The unit for each index is given at the top in square brackets. Three dots indicate where no index measurement is available.

Burstein, D., Faber, S. M., Gaskell, C. M., \& Krumm, N. 1984, ApJ, 287,586

Capaccioli, M., Cappellaro, E., Held, E. V., \& Vietri, M. 1993, A\&A, 274, 69

Carney, B. W., Latham, D. W., \& Laird, J. B. 1990, AJ, 99, 572

Davies, R. L., Sadler, E. M., \& Peletier, R. F. 1993, MNRAS, 262, 650

Dressler, A. 1980, ApJ, 236, 351

Dressler, A., Oemler, A. J., Couch, W. J., et al. 1997, ApJ, 490, 577

Edvardsson, B., Andersen, J., Gustafsson, B., et al. 1993, A\&A, 275, 101

Elson, R. A. W. 1997, MNRAS, 286, 771

Ferraro, F. R., D’Amico, N., Possenti, A., Mignani, R. P., \& Paltrinieri, B. 2001, ApJ, 561, 337 
Fisher, D., Franx, M., \& Illingworth, G. 1996, ApJ, 459, 110

Forbes, D. A., Beasley, M. A., Brodie, J. P., \& Kissler-Patig, M. 2001, ApJ, 563, L143

Forbes, D. A., Brodie, J. P., \& Grillmair, C. J. 1997, AJ, 113, 1652

González, J. J. 1993, Ph.D. Thesis

Hanes, D. A., \& Harris, W. E. 1986, ApJ, 304, 599

Horne, K. 1986, PASP, 98, 609

Kavelaars, J. J. 1998, PASP, 110, 758

Kissler-Patig, M., Richtler, T., Storm, J., \& della Valle, M. 1997, A\&A, 327, 503

Kundu, A., \& Whitmore, B. C. 1998, AJ, 116, 2841

Kuntschner, H. 2000, MNRAS, 315, 184

Kuntschner, H., Lucey, J. R., Smith, R. J., Hudson, M. J., \& Davies, R. L. 2001, MNRAS, 323, 615

Larsen, S. S., \& Brodie, J. P. 2002, AJ, 123, 1488

Larsen, S. S., Brodie, J. P., Beasley, M. A., \& Forbes, D. A. 2002, AJ, 124,828

Lee, H., Yoon, S., \& Lee, Y. 2000, AJ, 120, 998

Lee, J., \& Carney, B. W. 2002, AJ, 124, 1511

Maraston, C., \& Thomas, D. 2000, ApJ, 541, 126

McWilliam, A. 1997, ARA\&A, 35, 503

Michard, R., \& Marchal, J. 1994, A\&AS, 105, 481

Moehler, S., Landsman, W. B., \& Dorman, B. 2000, A\&A, 361, 937

Peletier, R. F. 1989, Ph.D. Thesis

Puzia, T. H., Saglia, R. P., Kissler-Patig, M., et al. 2002a, A\&A, 395, 45

Puzia, T. H., Zepf, S. E., Kissler-Patig, M., et al. 2002b, A\&A, 391, 453

Rich, R. M., Sosin, C., Djorgovski, S. G., et al. 1997, ApJ, 484, L25
Rosenberg, A., Saviane, I., Piotto, G., \& Aparicio, A. 1999, AJ, 118, 2306

Salaris, M., \& Weiss, A. 2002, A\&A, 388, 492

Schlegel, D. J., Finkbeiner, D. P., \& Davis, M. 1998, ApJ, 500, 525

Schroder, L. L., Brodie, J. P., Kissler-Patig, M., Huchra, J. P., \& Phillips, A. C. 2002, AJ, 123, 2473

Sharples, R. M., Zepf, S. E., Bridges, T. J., et al. 1998, AJ, 115, 2337

Silva, D. R., Boroson, T. A., Thompson, I. B., \& Jedrzejewski, R. I. 1989, AJ, 98, 131

Smith, R. J., Lucey, J. R., Hudson, M. J., Schlegel, D. J., \& Davies, R. L. 2000, MNRAS, 313, 469

Thomas, D., Greggio, L., \& Bender, R. 1999, MNRAS, 302, 537

Thomas, D., Maraston, C., \& Bender, R. 2002a, MNRAS, submitted

Thomas, D., Maraston, C., \& Bender, R. 2002b, in Reviews in Modern Astronomy No. 15, Astronomische Gesellschaft [astro-ph/0202166]

Tinsley, B. M. 1979, ApJ, 229, 1046

Tonry, J. L., Dressler, A., Blakeslee, J. P., et al. 2001, ApJ, 546, 681

Trager, S. C., Faber, S. M., Worthey, G., \& González, J. J. 2000, AJ, 119,1645

Trager, S. C., Worthey, G., Faber, S. M., Burstein, D., \& Gonzalez, J. J. 1998, ApJS, 116, 1

van Dokkum, P. G. 2001, PASP, 113, 1420

Vazdekis, A. 1999, ApJ, 513, 224

Worthey, G. 1994, ApJS, 95, 107

Worthey, G. 1998, PASP, 110, 888

Worthey, G., Faber, S. M., \& Gonzalez, J. J. 1992, ApJ, 398, 69

Worthey, G., \& Ottaviani, D. L. 1997, ApJS, 111, 377

Wyse, R. F. G., \& Gilmore, G. 1992, AJ, 104, 144 